\title{
Actuator-Work Concepts Applied to Unconventional Aerodynamic Control Devices
}

\author{
Christopher O. Johnston*, William H. Mason ${ }^{\dagger}$, Cheolheui Han ${ }^{\ddagger}$, Harry H. Robertshaw ${ }^{\S}$ and Daniel J. Inman ${ }^{* *}$ \\ Virginia Tech, Blacksburg, VA, 24060
}

This paper investigates the resistance to a change in wing shape due to the aerodynamic forces. In particular, the work required by an airfoil to overcome the aerodynamic forces and produce a change in lift is examined. The relationship between this work and the total aerodynamic energy balance is shown to have significant consequences for transient changes in airfoil shape. Specification of the placement of the actuators and the actuator energetics is shown to be required for the determination of the airfoil shape change requiring minimum energy input. A general, simplified actuator model is adopted in this study which assigns different values of actuator efficiency for negative and positive power output. Unsteady thin airfoil theory is used to analytically determine the pressure distribution and aerodynamic coefficients as a function of time for a ramp input of control deflection. This allows the required power and work to overcome the aerodynamic forces to be determined for a prescribed change in the airfoil camberline. The energy required for a pitching flat plate, conventional flap, conformal flap, and two variable camber configurations is investigated. For the pitching flat plate, the minimum energy pitching axis is shown to be dependent on the pitch rate and the initial angle of attack. The conformal flap is shown to require less actuator energy than the conventional flap to overcome the aerodynamic forces for a prescribed change in lift. The energy requirements of a variable camber configuration are shown to be sensitive to the layout of the variable camber device.

\section{Nomenclature}

$A_{n, b} \quad=$ Fourier coefficients defined in Eq. (3.6), $\mathrm{n}=1,2, . .$, and $b$ is the same as defined for $T_{a, b}$

$c \quad=$ chord length

$C_{L, n}=$ lift coefficient, $n=0,1$, and 2 correspond to the quasi-steady, apparent mass, and wake-effect terms

$C_{M, n} \quad=$ quarter-chord pitching moment coefficient, $n$ represents the terms defined with $C_{L}$

$C_{P} \quad=$ power coefficient for the power required to overcome the aerodynamic forces $(P)$

$C_{P a}=$ power coefficient for the required power input to the actuator $\left(P_{a}\right)$, (related to $C_{P}$ in Eq. (4.14))

$C_{W a} \quad=$ energy coefficient for the input energy required by an actuator $\left(W_{a}\right)$

$D=$ drag (the barred quantity represents the time-average)

$E \quad=$ energy dissipated to the wake per-unit time (the barred quantity represents the time-average)

$k \quad=$ ratio of the initial lift to the change in lift defined in Eqs. (5.17) and (6.2)

$P \quad=$ power required to overcome the aerodynamic forces (the barred quantity represents the time-average)

$P_{a} \quad=$ required power input to the actuator (related to $P$ in Eq. (2.7))

$Q_{n} \quad=$ defined in Eqs. $(4.8-4.13)$, where $n=1,2, \ldots, 5$

$q \quad=$ dynamic pressure

$t \quad=$ time

$t_{0} \quad=$ the time at which $P$ is zero (as shown in Figure 2.3)

$t^{*} \quad=$ time at the end of the unsteady motion

$T_{a, b}=$ components of the aerodynamic load distribution defined in Eqs. (3.12) and (3.17), $a=0$ and 1 corresponding to the quasi-steady and apparent mass terms, and $b=s$ and $d$ corresponding to the

\footnotetext{
* Graduate Research Assistant, Department of Aerospace and Ocean Engineering, Student Member AIAA

† Professor, Department of Aerospace and Ocean Engineering, Associate Fellow AIAA

* Visiting Scholar, Member AIAA

$\S$ Professor, Department of Mechanical Engineering

${ }^{* *}$ George R. Goodson Professor, Department of Mechanical Engineering, Fellow AIAA
} 
components resulting from the steady and damping boundary condition defined in Eq. (3.3) and (3.4)

$K_{a, b}=$ components of the lift coefficient defined in Eqs. (3.7) and (3.13), the subscripts are defined for $T_{a, b}$

$U=$ free-stream velocity

$w \quad=$ induced velocity on the airfoil camberline

$W \quad=$ work required to overcome the aerodynamic forces

$W_{a} \quad=$ required energy input to the actuator (related to $W$ in Eq. (2.8))

$x=$ distance along the airfoil chord aligned with the free-stream velocity

$x_{a} \quad=$ pitching axis

$\alpha \quad=$ angle of attack

$\beta=$ defines the time-history of the camberline shape change

$\chi \quad=$ load distribution function defined in Eq. (3.10)

$\delta \quad=$ Dirac delta function

$\Delta C_{p} \quad=$ unsteady pressure loading

$\gamma_{0}=$ quasi-steady vorticity distribution

$\eta \quad=$ actuator coefficient defined in Eq. (2.7)

$\theta \quad=$ polar coordinate along the airfoil surface which is related to $x$ in Eq. (3.6)

$\tau \quad=$ nondimensional time defined in Eq. (3.2b)

$\tau_{0} \quad=$ the value of $\tau$ at which $P$ (or $C_{P}$ ) is zero (nondimensional equivalent to $t_{0}$ defined in Figure 2.3)

$\tau^{*} \quad=$ nondimensional time which defines the end of the ramp input in Figure 4.1

$\psi \quad=$ shape function of the airfoil camberline defined in Eq. (3.2)

\section{Introduction}

$\mathrm{R}$ ECENT interest in morphing aircraft ${ }^{1,2}$ has initiated research concerning the characteristics of unconventional aerodynamic control devices. These unconventional, or morphing, devices are meant to provide an alternative to conventional hinged-flap configurations. For the design of a morphing device, it is desired to determine the change in wing shape that most efficiently produces the necessary change in the aerodynamic forces. Thus, understanding the process of producing a change in wing shape is of fundamental importance for morphing aircraft. One of the main design issues related to understanding this process is avoiding the weight penalty for unnecessary actuator capability. For a requested change in wing shape, the actuators on the wing must provide the work required to deform the wing while being acted on by the aerodynamic forces. Determining the change in wing shape that requires the minimum actuator work allows the morphing device to operate efficiently and with minimum actuator weight $\mathrm{w}^{3,4,5,6,7,8}$.

This paper presents a theoretical study of the relationship between the change in camberline shape of a twodimensional thin airfoil and the resistance of the aerodynamic forces to this change. This resistance will be represented by the work required from the actuators on the airfoil to overcome the aerodynamic forces while producing a change in camberline shape. The relationship between the output work produced by the actuators and the required input energy will be discussed and shown to affect the optimal changes in wing shape. A general actuator model will be presented and used throughout the analysis. A new method of unsteady thin airfoil theory for deforming camberlines will be presented and used for the energy calculation. This method allows the aerodynamic load distribution to be represented analytically, which provides insight into the work calculation. The energy required to produce a change in lift for a pitching flat plate will be thoroughly analyzed. The minimum energy pitching axes will be determined for various cases. The analysis of the pitching flat plate is applicable to variable twist morphing concepts. A comparison and analysis of the actuator energy cost for a conventional flap, conformal morphing flap, and two variable camber configurations will be presented. The analytic nature of this study clarifies the fundamental issues involved with the process of producing a change in airfoil shape.

\section{The Relationship between the Aerodynamic Energy Balance and Actuator Energy Cost}

For a wing moving in an inviscid potential flow, energy transfer between the wing and the fluid is achieved through the mechanical work required to produce wing motion or deformation while overcoming the fluid forces. This energy balance is stated mathematically through the following equation for conservation of energy, ${ }^{9}$

$$
P+D U=E
$$


where $P$ is the rate of work done by the wing against the fluid forces in a direction normal to the oncoming flow, $D$ is the drag force, $U$ is the free-stream velocity of the oncoming flow, and $E$ is the kinetic energy dissipated to the flow per unit time. For a thin airfoil in incompressible potential flow, the first two of these components are defined as follows ${ }^{10}$

$$
\begin{gathered}
P(t)=-\int_{0}^{c} \Delta p(x, t)\left[\frac{\partial z_{c}}{\partial t}(x, t)\right] d x \\
D(t)=-\int_{0}^{c} \Delta p(x, t)\left[\frac{\partial z_{c}}{\partial x}(x, t)\right] d x-S(t)
\end{gathered}
$$

where $\Delta p$ is the pressure loading on the airfoil, $z_{c}$ defines the camberline shape, and $S$ is the leading-edge suction force. Viscous effects may be included in the energy balance (Eq. 2.1) by including the skin friction component of drag in $D$ and viscous dissipation in $E .^{11}$

For the oscillatory motion of a thin airfoil, $\mathrm{Wu}^{12}$ shows that the average value of $E$ over a period of oscillation is always positive. $\mathrm{Wu}^{13}$ later explains that this point is readily apparent because in the frame of reference fixed to the undisturbed fluid, the kinetic energy of the basic flow is zero. Therefore, any unsteady motion of a body must increase the energy of the surrounding flow. It follows from Eq. (2.1) that for thrust to be generated from oscillatory airfoil motion, $\bar{P}$ must be positive. The case of $\bar{P}<0$ has a meaningful interpretation from two different points of view. The first point of view is that for an airfoil being propelled through a fluid. Although some energy is being taken from the flow (by definition of $\bar{P}<0$ ), more energy is being supplied to propel the airfoil (because $\bar{E}<0$, if $\bar{P}<0$, then from Eq. (2.1) $\bar{D}>-\bar{P}>0$ ). This case may be interpreted as flutter because the flow is supplying energy to the structure ${ }^{14}$. Patil ${ }^{15}$ points out that flutter analyses assume a constant flight speed, which is not practical because it implies that the aircraft propulsion system automatically accounts for the increase in drag caused by the unsteady wing motion. The second point of view is that of a fixed airfoil oscillating in an oncoming flow, which may be interpreted as the power extraction mode ${ }^{16,17}$. The difference between this case and the flutter case is that here there is no energy spent on propulsion because the oncoming flow, such as naturally occurring wind, provides the $\bar{D} U$ component of energy. It should be mentioned that the flutter mode can also be interpreted as a power extraction mode if the structure is designed for the task. The drawback is that the power spent on propulsion due to the oscillations will always be greater than the harvested power because $\bar{E}<0$.

For the transient motion or deformation of a thin airfoil, the consequences of the aerodynamic energy balance are significantly different from those of the oscillatory case discussed in the previous paragraph. The oscillatory case consists of a continuous motion that allows for a mean value over a period of oscillation to be defined. For the transient case, the unsteady motion ends at some prescribed time $\left(t^{*}\right)$ while the aerodynamic forces continue to change. This means that $P$ is zero after $t^{*}$, but the unsteady drag continues to act on the airfoil and therefore energy continues to be transferred to the wake. Notice that in the previous paragraph, no mention was made of the mean lift acting on the airfoil. This is because a constant aerodynamic force does not affect the mean energy balance of an oscillating airfoil ${ }^{18}$. For the transient case, though, a constant aerodynamic force component is significant. This significance is understood by recognizing that the energy required to produce a steady lifting flow from an initially non-lifting flow is infinite ${ }^{19}$. The reason for this infinite energy is shown by Lomax ${ }^{20}$ to be a result of the $1 / t$ dependence of the unsteady drag as $t$ tends to infinity. With an initial value of lift acting on an airfoil during a transient motion, the flow has the ability to transfer some of the infinite energy present initially in the flow to the airfoil. If the initial lift on the airfoil is zero, a result analogous to $\mathrm{Wu}$ 's result that $\bar{E}>0$ may be stated as follows: if the fluid is undisturbed at $t=0$, then

$$
\int_{0}^{t} E(t) d t>0
$$

For an airfoil with a finite value of lift at $t=0$, this inequality does not necessarily hold. Another consequence of the infinite energy required to produce a change in lift is that it makes any attempt to minimize the energy lost in the wake for a given change in lift invalid. Recognizing that an infinite amount of time is required for the unsteady drag to transfer the infinite energy to the flow, it becomes clear that the addition of a steady component of drag (e.g. 
viscous or 3-D induced drag) will also require an infinite amount of energy to overcome. Adding the practical consideration that these steady components of drag will overshadow the unsteady component of drag for most values of time, it becomes clear that the unsteady drag will be an insignificant component of the energy required by an aircraft propulsion system. On the other hand, the power required to overcome the aerodynamic forces and produce camberline deformations $(P)$, which is finite, is not affected by the addition of steady drag components. Therefore, the component $P$ drives the design of the actuation systems on an aircraft that produce camberline deformations. The remainder of this paper will be concerned with the determination and minimization of the energy required to produce camberline deformations; with it being accepted from the practical standpoint mentioned above that the infinite energy required to overcome the unsteady component of drag is being ignored.

Figure 2.1 shows one way of allocating the total required actuator power $\left(P_{\text {out }}\right)$ for a general airfoil control device. The structural forces would be present on any morphing-type device that must deform an outer skin. Frictional forces may also be grouped in the structural forces category, which would also apply to conventional hinged flaps. The inertial forces are present for any device, but are negligible compared to the aerodynamic and structural forces. As previously stated, the current study is concerned with the power required to overcome the aerodynamic forces $(P)$, and therefore $P_{\text {out }}$ is assumed to equal $P$ in Figure 2.1. For a prescribed change in camberline shape along a defined path between $t=0$ and $t=t^{*}$, the total energy required to overcome the aerodynamic forces is defined as

$$
W=\int_{0}^{t^{*}} P(t) d t
$$

The power required by the actuator to produce $P$ is defined as $P_{a}$ in Figure 2.1. The corresponding energy input to the actuators for a prescribed camberline deformation is defined as

$$
W_{a}=\int_{0}^{t^{*}} P_{a}(t) d t
$$

Note that Eq. (2.5) and (2.6) are defined separately for each control surface or actuator on the airfoil. The value of $P$ required for each control surface or actuator is distinguished by the $d z_{c} / d t$ term in Eq. (2.2).

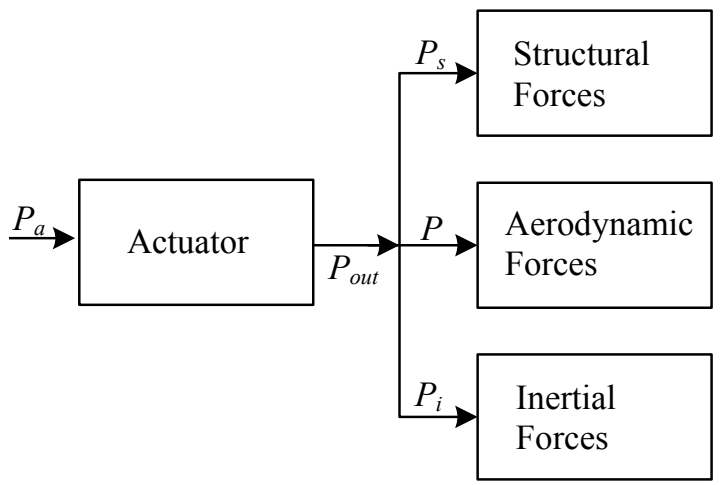

Figure 2.1. The distribution of the provided actuator power for a general configuration.

To obtain the quantity $P_{a}$, knowledge of the actuator energetics and actuator placement is required. For the current study, which is intended to investigate the fundamentals of the actuator energy required to overcome the aerodynamic forces, a general model of the actuator energetics is proposed. The model is defined as follows

$$
\begin{aligned}
& \text { for } P_{\text {out }} \geq 0, P_{a}=P_{\text {out }} \\
& \text { for } P_{\text {out }}<0, P_{a}=\eta\left|P_{\text {out }}\right|
\end{aligned}
$$

where $\eta$ is a constant ranging from -1 to 1 depending on the actuator. A separate efficiency could be defined for positive values of $P_{\text {out }}$ (so that $100 \%$ actuator efficiency is not assumed), although this implies just multiplying $W_{a}$ by a constant (since $\eta$ will change accordingly). This will not influence a comparison between different control 
surface configurations and is therefore not used for this analysis. Figure 2.2 illustrates Eq. (2.7) for three key values of $\eta$. For $\eta=1$, the actuator requires the same power input to produce negative values of $P_{\text {out }}$ as it does to produce positive values. Recall that positive $P_{\text {out }}$ values indicate that the actuator motion is resisted by the external forces while negative values indicate that the external forces act in the direction of actuator motion. For $\eta=0$, the actuator requires no power input and allows no power to be extracted while producing negative values of $P_{\text {out }}$. This case is the most consistent with feedback controlled pneumatic ${ }^{21}$ and hydraulic ${ }^{22}$ actuators, which require only the controlled release of pressurized fluid to produce negative power. The neglecting of negative work values has also been considered for the energy-cost analysis of insect flight ${ }^{23}$ and human muscles ${ }^{24}$. The $\eta=-1$ case allows the actuator to store the incoming energy associated with negative values of $P_{\text {out }}$ to be used later to produce positive $P_{\text {out }}$ values with $100 \%$ conversion efficiency. This value of $\eta$ allows $W_{a}$ to be negative and zero for certain cases.

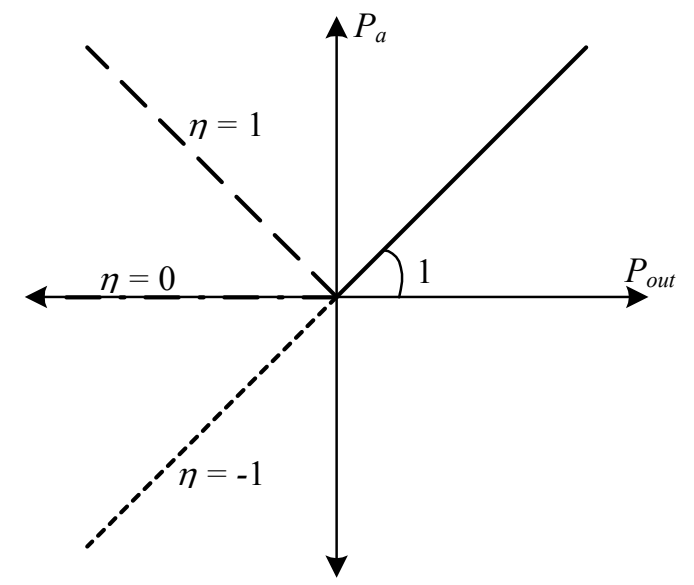

Figure 2.2. The relationship between $\boldsymbol{P}_{\text {out }}$, the required rate of actuator work, and $\boldsymbol{P}_{a}$, the rate of actuator energy, for the proposed general actuator model.

Applying the general actuator model of Eq. (2.7) to Eq. (2.6), the equation for the total required actuator energy input can be written as

$$
W_{a}=W_{+}+\eta W_{-}
$$

where $W_{+}$and $W_{\text {. }}$ are the absolute values of the positive and negative components of the integral in Eq. (2.6). An example of these components is shown in Figure 2.3, where in this case $W_{+}$is the integral of $P$ from $t=0$ to $t_{0}$ and $W$. is the negative of the integral from $t_{0}$ to $t^{*}$.

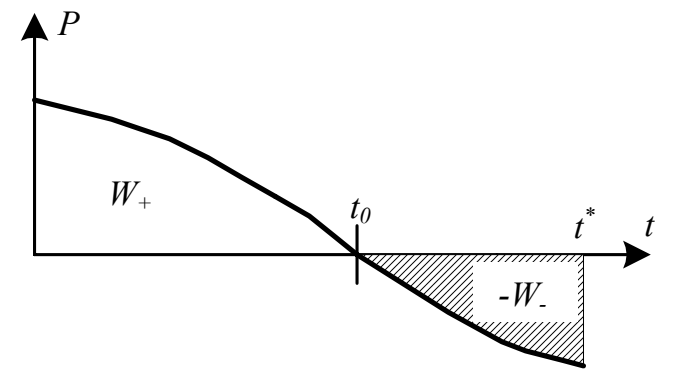

Figure 2.3. An example of the separation of $W$ into $W_{+}$and $W$ - components for a given transient motion.

\section{Unsteady Thin Airfoil Theory Applied to Control Surface Motions}

The prediction and understanding of the unsteady aerodynamic forces resulting from a change in camberline shape are necessary to analyze the energy characteristics of a deforming camberline. This section presents a brief description of unsteady thin airfoil theory in a form convenient for the analysis of general deforming camberlines. The presentation of this material is necessary because there are only a few past studies on the unsteady pressure distribution $^{25,26,27}$, which is required for the calculation of $P$ in Eq. (2.2). Previous research on the unsteady pressure distribution has all been focused on the oscillatory case, and the extension to the transient case has not been discussed. The method presented here follows directly from steady thin airfoil theory, therefore allowing a physical 
interpretation of each of the unsteady components. A more detailed discussion of this approach can be found in Han, et al. ${ }^{28}$

Following the von Karman and Sears ${ }^{29}$ approach to unsteady thin airfoil theory, the aerodynamic forces acting on an unsteady airfoil can be separated into three components: quasi-steady, apparent mass, and wake-effect. The quasi-steady component represents the solution of the steady thin airfoil problem to the instantaneous boundary conditions. The term "instantaneous boundary condition" is used instead of "instantaneous camberline shape" because an extra component of induced velocity is caused by the rate-of-change of the camberline shape. The component of the aerodynamic force resulting from this induced velocity usually ${ }^{\dagger \dagger}$ damps the unsteady motion, and will therefore be referred to as the aerodynamic damping. Figures 3.1 and 3.2 illustrate the steady and damping components of the quasi-steady boundary condition for a pitching flat-plate and a deflecting flap. Defining the steady component as $w_{s}$ and the damping component as $w_{d}$, the total boundary condition can be written as

$$
w=w_{s}+w_{d}
$$

From Eq. (3.1), the quasi-steady aerodynamic forces can be derived separately for the steady and damping terms.
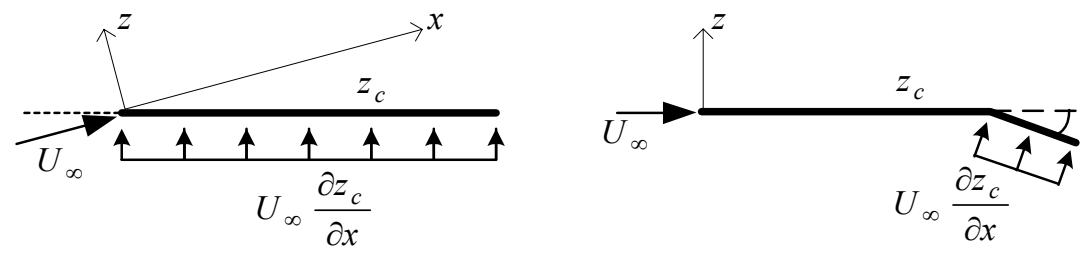

Figure 3.1. The steady terms of the boundary condition for a pitching airfoil and a deflecting flap.
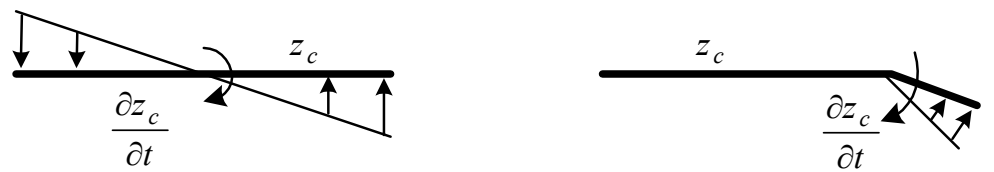

Figure 3.2. The damping terms of the boundary condition for a pitching airfoil and a deflecting flap.

It is convenient to restrict the present analysis to time-dependent camberlines of the following form

$$
z_{c}(x, \tau)=\psi(x) \beta(\tau)
$$

where $\psi$ defines the shape of camberline (for example a flapped or a parabolic camberline), and $\beta$ defines the time varying magnitude of the camberline (for example the flap deflection angle or magnitude of maximum camber). Also, let $\tau$ represent a nondimensional time, defined as

$$
\tau=\frac{U t}{c}
$$

Note that Eq. (3.2) cannot represent shapes such as a time varying flap-to-chord-ratio because $\psi$ is not a function of time. From Eq. (3.2), the steady and damping terms of Eq. (3.1) can be written as

$$
\begin{aligned}
& w_{s}=\frac{\partial \psi}{\partial x} \beta \\
& w_{d}=\frac{\psi}{c} \beta^{\prime}
\end{aligned}
$$

where $\beta$ is defined as $d \beta / d \tau$.

\section{A. The Quasi-Steady Terms}

As mentioned previously, the quasi-steady component of the aerodynamic force is the component resulting from steady thin airfoil theory. While the quasi-steady force coefficients for various camberline shapes are well known,

\footnotetext{
${ }^{\dagger \dagger}$ For all practical camberline configurations except for leading edge flaps this is true.
} 
for example a conventional hinged leading edge ${ }^{30}$ and trailing edge ${ }^{31}$ flap and an NACA 4-digit camberline $\mathrm{e}^{32}$, there are few analytic quasi-steady pressure distributions presented in the literature (most notable is Allen' ${ }^{33}$ solution for a trailing edge flap).

It is convenient to represent the quasi-steady airfoil vorticity $\left(\gamma_{0}\right)$ using Glauert's Fourier series ${ }^{34}$, which is written as

$$
\gamma_{0}(\theta)=2 U\left(A_{0}\left[\frac{1+\cos \theta}{\sin \theta}\right]+\sum_{n=1}^{\infty} A_{n} \sin n \theta\right)
$$

where

$$
\begin{gathered}
x=\frac{c}{2}(1-\cos \theta) \\
A_{0}=-\frac{1}{\pi} \int_{0}^{\pi} w(\theta, t) d \theta \\
A_{n}=\frac{2}{\pi} \int_{0}^{\pi} w(\theta, t) \cos (n \theta) d \theta
\end{gathered}
$$

The Fourier coefficients in Eq. (3.6) may be separated into steady and damping terms $\left(A_{0, s}, A_{0, d}, A_{n, s}\right.$, and $\left.A_{n, d}\right)$ by using $w_{s}$ or $w_{d}$ in place of $w$. The quasi-steady lift $\left(C_{L, 0}\right)$ and quarter-chord pitching moment $\left(C_{M, 0}\right)$ are written in terms of the Fourier coefficients as

$$
\begin{aligned}
C_{L, 0}= & \pi\left(2 \bar{A}_{0, s}+\bar{A}_{1, s}\right) \beta+\pi\left(2 \bar{A}_{0, d}+\bar{A}_{1, d}\right) \beta^{\prime} \\
= & K_{0, s} \beta+K_{0, d} \beta^{\prime} \\
C_{M, 0} & =\frac{\pi}{4}\left(\bar{A}_{2, s}-\bar{A}_{1, s}\right) \beta+\frac{\pi}{4}\left(\bar{A}_{2, d}-\bar{A}_{1, d}\right) \beta^{\prime} \\
& =J_{0, s} \beta+J_{0, d} \beta^{\prime}
\end{aligned}
$$

where the bar over the Fourier coefficients indicates that they are per-unit $\beta$ or $\beta$. Similarly, from Eq. (3.5) the quasi-steady load distribution is written as (recall that $\Delta C_{p, 0}=2 \gamma_{0} / U$ )

$$
\begin{aligned}
\Delta C_{p, 0}(\theta) & =\left(4 \bar{A}_{0, s}\left[\frac{1+\cos \theta}{\sin \theta}\right]+4 \sum_{n=1}^{\infty} \bar{A}_{n, s} \sin n \theta\right) \beta+\left(4 \bar{A}_{0, d}\left[\frac{1+\cos \theta}{\sin \theta}\right]+4 \sum_{n=1}^{\infty} \bar{A}_{n, d} \sin n \theta\right) \beta^{\prime} \\
& =\left(\bar{A}_{0, s} \chi(\theta)+T_{0, s}(\theta)\right) \beta+\left(\bar{A}_{0, d} \chi(\theta)+T_{0, d}(\theta)\right) \beta^{\prime}
\end{aligned}
$$

where

$$
\begin{aligned}
\chi(\theta) & =4\left[\frac{1+\cos \theta}{\sin \theta}\right] \\
T_{0, s}(\theta) & =4 \sum_{n=1}^{\infty} \bar{A}_{n, s} \sin n \theta \\
T_{0, d}(\theta) & =4 \sum_{n=1}^{\infty} \bar{A}_{n, d} \sin n \theta
\end{aligned}
$$

Allen $^{35}$ showed that the infinite series in Eq. (3.10) may be written in terms of the following integral 


$$
\sum_{n=1}^{\infty} A_{n} \sin n \theta=\frac{1}{\pi} \int_{0}^{\pi} \frac{w\left(\theta_{0}, t\right) \sin \theta}{\cos \theta_{0}-\cos \theta} d \theta_{0}
$$

Thus, the $T_{0}$ values in Eq. (3.10) may be rewritten as

$$
\begin{aligned}
& T_{0, s}(\theta)=\frac{4}{\pi} \int_{0}^{\pi} \frac{\frac{\partial \psi}{\partial x}\left(\theta_{0}\right) \sin \theta}{\cos \theta_{0}-\cos \theta} d \theta_{0} \\
& T_{0, d}(\theta)=\frac{4}{\pi} \int_{0}^{\pi} \frac{\psi\left(\theta_{0}\right) \sin \theta}{\cos \theta_{0}-\cos \theta} d \theta_{0}
\end{aligned}
$$

From Eqs. (3.6), (3.9), and (3.12), the quasi-steady load distribution may be determined analytically for a given $\psi$. It will be shown that the quasi-steady components are required as inputs for the calculation of the apparent mass and wake-effect terms.

\section{B. The Apparent Mass Terms}

The apparent mass lift and pitching moment coefficients are defined $\mathrm{as}^{26}$

$$
\begin{gathered}
C_{L, 1}=\frac{\partial}{\partial \tau}\left(\frac{1}{2 U_{\infty}} \int_{0}^{\pi} \gamma_{0}(\theta) \cos \theta \sin \theta d \theta\right) \\
C_{M, 1}=\frac{\partial}{\partial \tau}\left(\int_{0}^{\pi} \frac{1}{8 U_{\infty}} \gamma_{0}(\theta)\left[\cos ^{2} \theta-1 / 2-\cos \theta\right] \sin \theta d \theta\right)
\end{gathered}
$$

These expressions can be written in terms of steady and damping Fourier coefficients defined in Eq. (3.6) as follows

$$
\begin{gathered}
C_{L, 1}=\frac{\pi}{4}\left(2 \bar{A}_{0, s}+\bar{A}_{2, s}\right) \beta^{\prime}+\frac{\pi}{4}\left(2 \bar{A}_{0, d}+\bar{A}_{2, d}\right) \beta^{\prime \prime} \\
=K_{1, s} \beta^{\prime}+K_{1, d} \beta^{\prime \prime} \\
C_{M, 1}=-\frac{\pi}{32}\left(4 \bar{A}_{0, s}+\bar{A}_{1, s}+2 \bar{A}_{2, s}-\bar{A}_{3, s}\right) \beta^{\prime}-\frac{\pi}{32}\left(4 \bar{A}_{0, d}+\bar{A}_{1, d}+2 \bar{A}_{2, d}-\bar{A}_{3, d}\right) \beta^{\prime \prime} \\
=J_{1, s} \beta^{\prime}+J_{1, d} \beta^{\prime \prime}
\end{gathered}
$$

where $\beta$ ' represents $d^{2} \beta / d \tau^{2}$. The apparent mass pressure distribution is determined using Neumark's ${ }^{36}$ approach. After some manipulation of Neumark's equations, which require the separation of $\gamma_{0}$ into circulatory and noncirculatory components, the following equation is obtained ${ }^{25}$

$$
\begin{aligned}
\Delta C_{p, 1} & =\left(2 \bar{A}_{0, s} \sin \theta-\bar{A}_{1, s} \theta+\frac{1}{2} \int_{0}^{\theta} T_{0, s}(\theta) \sin \theta d \theta\right) \beta^{\prime}+\left(2 \bar{A}_{0, d} \sin \theta-\bar{A}_{1, d} \theta+\frac{1}{2} \int_{0}^{\theta} T_{0, d}(\theta) \sin \theta d \theta\right) \beta^{\prime \prime} \\
& =T_{1, s}(\theta) \beta^{\prime}+T_{1, d}(\theta) \beta^{\prime \prime}
\end{aligned}
$$

As mentioned previously, the apparent mass terms are dependent upon the quasi-steady terms. The terms $T_{l, s}$ and $T_{l, d}$ represent the most difficult components to obtain analytically because they depend upon the integration of $T_{0, s}$ and $T_{0, d}$. It is convenient to recognize that for a camberline defined by Eq. (3.2a), the component $T_{0, d}$ is identical to $T_{1, s}{ }^{25}$. 


\section{The Wake-Effect Terms}

For arbitrary time-dependent quasi-steady lift variations, the wake-effect lift and pressure distribution are obtained using a superposition of step input responses. The lift response to a step input in $C_{L, 0}$ is referred to as the Wagner function $(\phi)^{37}$. Linear superposition of the Wagner function is achieved using the Duhamel integral ${ }^{38}$, which allows the wake-effect lift $\left(C_{L, 2}\right)$ to be written as

$$
C_{L, 2}(\tau)=C_{L, 0}\left(\tau_{0}\right) \phi(\tau)+\int_{\tau_{0}}^{\tau} \frac{d C_{L, 0}}{d \sigma}(\sigma) \phi(\tau-\sigma) d \sigma
$$

Neumark ${ }^{36}$ shows that $\Delta C_{p, 2}$ has exactly the same $\theta$-dependence as a $\Delta C_{p, 0}$ produced by an angle of attack. Thus, the equation for the wake-effect pressure coefficient $\left(\Delta C_{p, 2}\right)$ can be written as

$$
\Delta C_{p, 2}(\tau)=\frac{2}{\pi c}\left[\frac{1+\cos \theta}{\sin \theta}\right] C_{L, 2}(\tau)
$$

The Wagner function, shown in Figure 3.3, can be approximated as ${ }^{39}$

$$
\phi(\tau)=-0.165 e^{-0.091 \tau}-0.335 e^{-0.6 \tau}
$$

Also shown in Figure 3.3 is the result of the Duhamel integral in Eq. (3.18) for a ramp input that terminates at $\tau^{*}$. This result for a ramp input will be used in later sections of this paper.

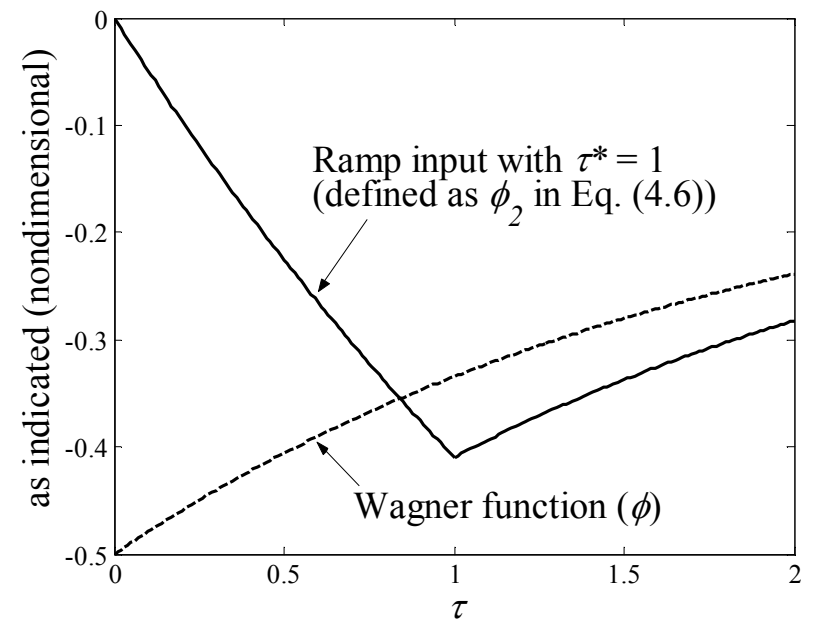

Figure 3.3. Illustration of the Wagner function and the function obtained from the evaluation of the integral in Eq. (3.18) for a ramp input.

For a sudden step change in circulation (due to a step change in angle of attack, flap deflection, etc.), the total lift coefficient can be written as

$$
C_{L}(\tau)=C_{L, 0}+\phi(\tau) C_{L, 0}+C_{L, 1}(\tau)
$$

where the combination of $\phi$ and $C_{L, 0}$ is $C_{L, 2}$. The apparent mass lift for a step change in $C_{L, 0}$ is written using the Dirac delta function to represent the time-derivative in Eq. (3.13) as follows

$$
C_{L, 1}=\frac{\delta(\tau)}{4} \pi\left(2 A_{0}+A_{2}\right)
$$

Recall that the Dirac delta function has the following properties 


$$
\begin{aligned}
& \delta(\tau=0)=\infty \\
& \delta(\tau \neq 0)=0 \\
& \int_{-\infty}^{\infty} \delta(\tau) d \tau=1
\end{aligned}
$$

The last property in Eq. (3.23) will be important when discussing the work required to overcome the aerodynamic forces. The load distribution of this apparent mass pulse is obtained by exchanging the Dirac delta function for the time derivative of $\beta$ in Eq. (3.17).

To conclude this section, Figure 3.4 illustrates the components of the unsteady load distribution for a conventional hinged flap with a flap-to-chord ratio of 0.25 . These terms are determined analytically from Eqs. (3.9) and (3.17). Note that the time-dependent magnitude of these components, as indicated by Eqs. (3.9) and (3.17), depend on $\beta$ and its first and second derivative with respect to $\tau$. For a ramp input of $\beta$, the quasi-steady term from the steady boundary condition, component (a), varies linearly with $\beta$ while the damping quasi-steady term, component (b), and the apparent mass term from the rate-of-change of component (a), component (c), are constant with time. The wake-effect component, which has the shape of an angle of attack load distribution, is not shown because its magnitude depends on the time-history of $\beta$. From this figure it is seen that if $\beta^{\prime}$ and $\beta^{\prime \prime}$ have the same sign as $\beta$, then the hinge moment coefficient of the flap is increased above the steady value.

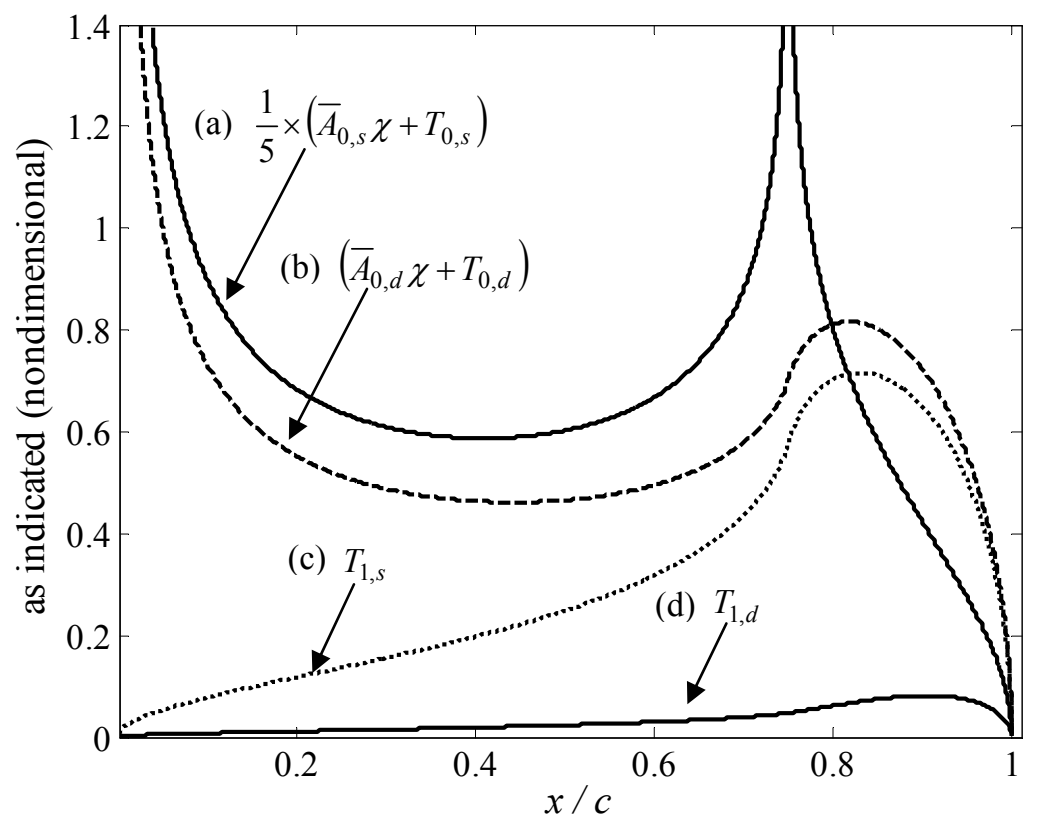

Figure 3.4. The components of the unsteady load distribution for a conventional hinged flap with a flap-to-chord ratio of 0.25 .

\section{The Aerodynamic Work for a Ramp Input of Control Deflection}

For a camberline defined by Eq. (3.2), the time-dependence of the camberline deformation is defined entirely by the function $\beta$. This section will derive the aerodynamic work and power components discussed in Section II for the function $\beta$ defined as a terminated ramp, which will be written as

$$
\begin{gathered}
\beta(\tau)=\bar{\beta}_{0}, \quad-\infty<\tau<0 \\
\beta(\tau)=\bar{\beta}_{0}+\frac{\tau}{\tau^{*}} \Delta \bar{\beta}, \quad 0 \leq \tau \leq \tau * \\
\beta(\tau)=\bar{\beta}_{0}+\Delta \bar{\beta}, \quad \tau^{*}<\tau<\infty
\end{gathered}
$$


where $\bar{\beta}_{0}$ is the initial value of $\beta$, and $\Delta \bar{\beta}$ is the change in $\beta$ between $\tau=0$ and $\tau=\tau^{*}$. These terms are illustrated in Figure 4.1 along with the corresponding first and second derivatives of $\beta$. Notice that the second derivate is defined by two Dirac delta function impulses.

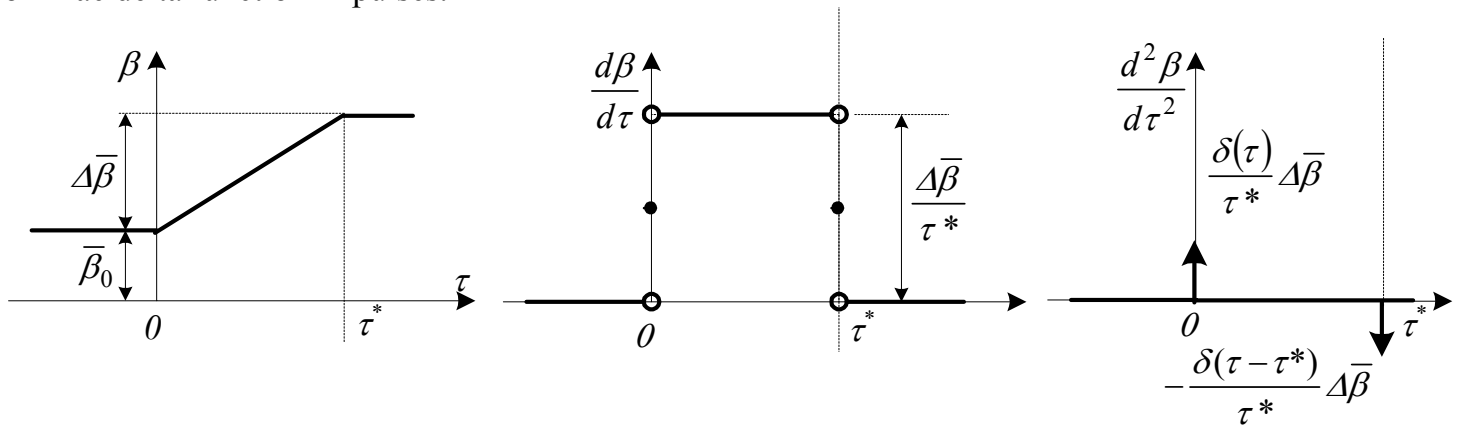

Figure 4.1. The specified time-history of the camberline deformation $\beta$ and the corresponding time-derivatives.

The power required to overcome the aerodynamic forces was defined in Eq. (2.2). It will be convenient to represent the power by the following nondimensional power coefficient $\left(C_{P}\right)$

$$
\begin{aligned}
C_{P}(\tau) & =\frac{P}{q U c} \\
& =-\frac{1}{c^{2}} \int_{0}^{c} \Delta C_{p}(x, \tau)\left[\frac{\partial z_{c}}{\partial \tau}(x, \tau)\right] d x
\end{aligned}
$$

Collecting the quasi-steady, apparent mass, and wake effect components of the aerodynamic force from the previous section, $\Delta C_{p}$ may be written in terms of $\beta$ as follows

$$
\Delta C_{p}(x, t)=\alpha_{u n}(t) \chi(x)+\left(\bar{A}_{0, s} \chi(x)+T_{0, s}(x)\right) \beta(t)+\left(\bar{A}_{0, d} \chi(x)+T_{0, d}(x)+T_{1, s}(x)\right) \beta^{\prime}(t)+T_{1, d}(x) \beta^{\prime \prime}(t)
$$

For the $\beta$ defined in Eq. (4.1), the wake effect term, $\alpha_{u n}$, evaluates to the following

$$
\alpha_{u n}(\tau)=\frac{\Delta \bar{\beta}}{2 \pi \tau^{*}}\left[K_{0, d} \phi_{1}(\tau)+K_{0, s} \phi_{2}(\tau)\right]
$$

where

$$
\begin{gathered}
\phi_{1}(\tau) \equiv \phi(\tau) \\
=-0.165 e^{-0.091 \tau}-0.335 e^{-0.6 \tau} \\
\phi_{2}(\tau) \equiv \int_{0}^{\tau} \phi(\tau-\sigma) d \sigma \\
=-2.37152+0.55833 e^{-0.6 \tau}+1.81319 e^{-0.091 \tau}
\end{gathered}
$$

Substituting $\Delta C_{p}$ from Eqs. (4.3 - 4.6) and $z_{c}$ from Eqs. (3.2 and 4.1) into Eq. (4.2) allows the power coefficient for a single control surface to be written as

$$
C_{P}=\frac{\Delta \bar{\beta}^{2}}{\tau^{* 2}}\left[Q_{1} \phi_{1}(\tau)+Q_{2} \phi_{2}(\tau)+Q_{3} \tau+Q_{4}+\left(\delta(\tau)-\delta\left(\tau-\tau^{*}\right)\right) Q_{5}\right]+\frac{\Delta \bar{\beta} \bar{\beta}_{0}}{\tau^{*}} Q_{3}
$$

where the $Q$ terms are defined as 


$$
\begin{gathered}
Q_{1}=-\frac{K_{0, d}}{2 \pi} \int_{0}^{c} \psi(x) d x \\
Q_{2}=-\frac{K_{0, s}}{2 \pi} \int_{0}^{c} \psi(x) d x \\
Q_{3}=-\int_{0}^{c}\left(\bar{A}_{0, s} \chi(x)+T_{0, s}(x)\right) \psi(x) d x \\
Q_{4}=-\int_{0}^{c}\left(\bar{A}_{0, d} \chi(x)+2 T_{0, d}(x)\right) \psi(x) d x \\
Q_{5}=-\frac{1}{2} \int_{0}^{c} T_{1, d}(x) \psi(x) d x
\end{gathered}
$$

Note that the $1 / 2$ in the $Q_{5}$ equation is a result of the definition of $d \beta / d \tau$ at $\tau=0$ and $\tau^{*}$, which from Figure 4.1 can be written as

$$
\begin{aligned}
\frac{d \beta}{d \tau}(\tau=0) & =-\frac{d \beta}{d \tau}\left(\tau=\tau^{*}\right) \\
& =\frac{1}{2} \frac{\Delta \bar{\beta}}{\tau^{*}}
\end{aligned}
$$

For linear camberline shapes, $\psi$ is linear, and each term in Eq. (4.7) may be interpreted as a component of the dynamic hinge-moment coefficient multiplied by the flap deflection rate $(d \beta / d \tau)$. The $Q_{1}$ and $Q_{2}$ terms are due to the wake effect forces, $Q_{3}$ is due to the quasi-steady forces, and $Q_{4}$ and $Q_{5}$ are due to the apparent mass forces. The Dirac delta functions in Eq. (4.7) are a result of the acceleration pulse of the camberline as shown in Figure 4.1.

Having obtained an expression for the output power required by an actuator to overcome the aerodynamic forces during a ramp input of camberline deformation, the input energy required by the actuator $\left(W_{a}\right)$ may be calculated using Eqs. (2.6 - 2.8). The nondimensional input energy coefficient is defined as

$$
\begin{aligned}
C_{W a} & =\frac{W_{a}}{q c^{2}} \\
& =\int_{0}^{\tau^{*}} C_{P_{a}} d \tau
\end{aligned}
$$

where $C_{P a}$ is defined through the general actuator model defined in Eq. (2.7), which can be written in terms of $C_{P}$ as

$$
\begin{aligned}
& \text { for } C_{P} \geq 0, C_{P a}=C_{P} \\
& \text { for } C_{P}<0, C_{P a}=\eta\left|C_{P}\right|
\end{aligned}
$$

From Eq. (4.14), the integration required by Eq. (4.13) for $C_{W a}$ can be separated into positive $\left(C_{W^{+}}\right)$and negative $\left(C_{W-}\right)$ components as follows

$$
C_{W a}=C_{W+}+\eta C_{W-}
$$

which is equivalent to Eq. (2.8) and is illustrated in Figure 2.3. Note that the two Dirac delta functions in Eq. (4.7) result in there always being both a component of $C_{W+}$ and $C_{W-}$ present. Assuming $Q_{5}$ is greater than zero, the impulses at $\tau=0$ and $\tau=\tau^{*}$ provide components of $C_{W+}$ and $C_{W-}$, respectively. These components can both be written as 


$$
C_{W, \delta}=\frac{\Delta \bar{\beta}^{2}}{\tau^{* 2}} Q_{5}
$$

which represent the instantaneous transfer of energy from the airfoil to the surrounding fluid. Although this is an unrealistic concept, it is accepted because it simplifies the effect of camberline acceleration by concentrating it at the beginning and end of the unsteady motion.

The difficulty in applying Eq. (4.15) is that the integrations required for $C_{W^{+}}$and $C_{W \text { - }}$ can only be evaluated analytically for special cases. The reason for this is that $\tau_{0}$ must be found and then used as a limit of integration for the evaluation of $C_{W^{+}}$and $C_{W-}\left(\tau_{0}\right.$ is equivalent to $t_{0}$ in Figure 2.3). The analytic evaluation of $\tau_{0}$ is made difficult by the exponentials present in Eqs. (4.5) and (4.6). For Eq. (4.15) to be evaluated analytically, $\tau_{0}$ must be less than zero or greater than $\tau^{*}$ so that $C_{P}$ remains either positive or negative throughout the deformation process. Details of these considerations are explained most effectively through an example, which is the focus of the next section.

\section{Application to a Pitching Flat-Plate Airfoil}

The application of the actuator energy theory developed in the previous sections to a pitching flat-plate identifies many of the interesting aspects of the theory. Consider the flat plate shown in Figure 5.1. The shape function of Eq. (3.2) is simply

$$
\psi(x)=x_{a}-x
$$

and the time dependent angle of attack, $\beta(\tau)=\alpha(\tau)$, is specified to be the ramp input defined in Eq. (4.1).

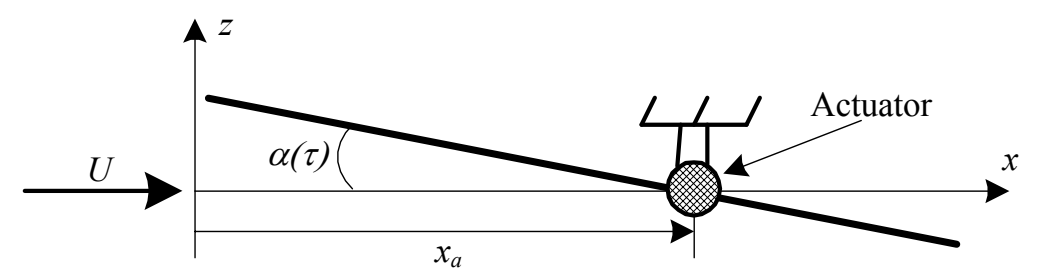

Figure 5.1. Definition of the geometry and actuator placement for a pitching flat plate.

The $Q$ terms from Eq. (4.8 - 4.13) evaluate to the following

$$
\begin{gathered}
Q_{1}=\pi\left(\frac{3}{8}-2 x_{a}+2 x_{a}^{2}\right) \\
Q_{2}=Q_{3}=\frac{\pi}{2}\left(1-4 x_{a}\right) \\
Q_{4}=\pi\left(\frac{3}{4}-\frac{5}{2} x_{a}+2 x_{a}^{2}\right) \\
Q_{5}=\frac{\pi}{2}\left(\frac{9}{64}-\frac{1}{2} x_{a}+\frac{1}{2} x_{a}^{2}\right)
\end{gathered}
$$

Applying these functions to Eq. (4.7) for a value of $x_{a} / c=0.5$, the time-history of $C_{P}$ was determined for various values of $\tau^{*}$ and is plotted in Figure 5.2. The axes of Figure 5.2 are normalized with $\tau^{*}$ to allow the various cases to be shown on the same figure. This figure shows that the required positive work $\left(C_{W^{+}}\right)$decreases as $\tau^{*}$ increases, which is a result of reduced aerodynamic damping. Because the initial angle of attack $\left(\alpha_{0}\right)$ is zero for this case, Eq. (4.7) indicates that the value of $\tau$ at which $C_{P}$ is zero $\left(\tau_{0}\right)$ is independent of $\tau^{*}$ (this is not obvious in Figure 5.2 because of the scaling of the axes). 


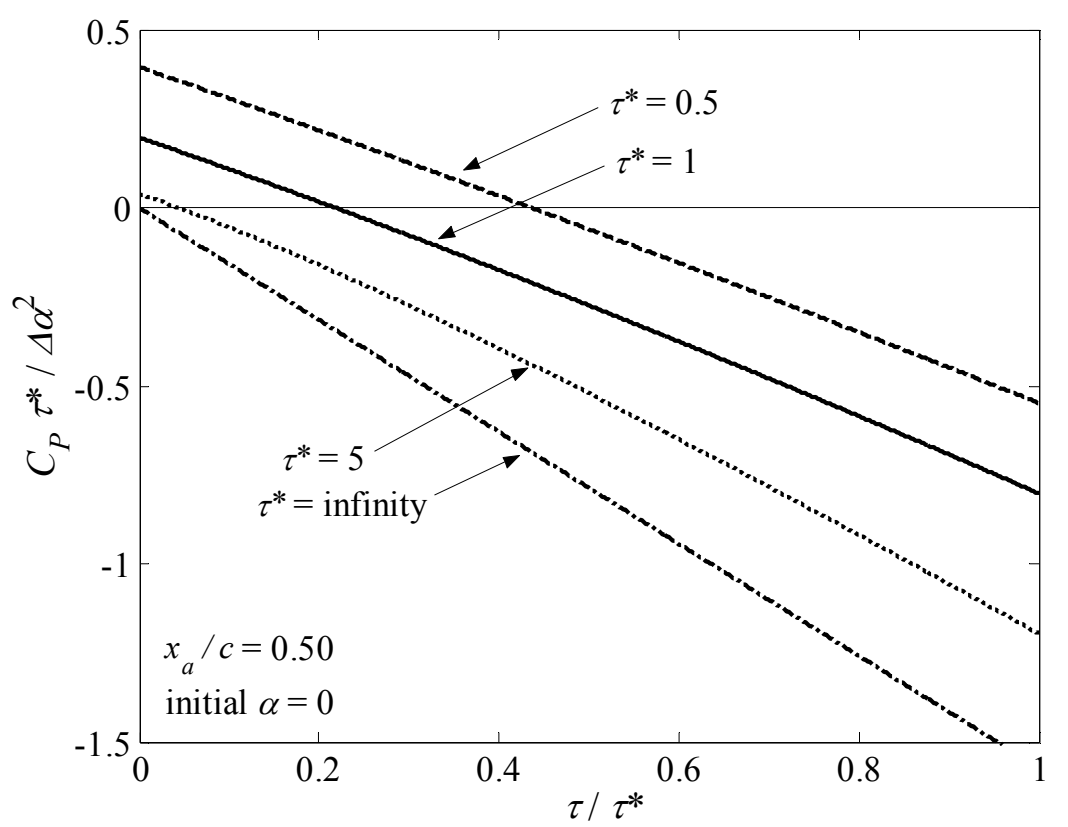

Figure 5.2. The time-history of the power coefficient for a ramp input of $\alpha$ for various values of $\tau^{*}$

It turns out that this initially nonlifting case allows for the approximate analytic evaluation of $C_{W^{+}}$and $C_{W-\text {. This }}$ is possible because $\tau_{0}$ may be determined analytically by making use of a few valid assumptions. The solution process for $\tau_{0}$ is initiated by setting $C_{P}$ from Eq. (4.7) equal to zero,

$$
Q_{1} \phi_{1}\left(\tau_{0}\right)+Q_{2} \phi_{2}\left(\tau_{0}\right)+Q_{3} \tau_{0}+Q_{4}=0
$$

where $\phi_{1}$ and $\phi_{2}$ are defined in Eqs. (4.5) and (4.6). It is observed in Figure 5.2 that $\tau_{0}$ is less than one, which is true for values of $x_{a} / c>0.45$. It is also observed from Eqs. (4.5) and (4.6) that the coefficients in the exponentials are less than one. From these observations it is concluded that $\phi_{1}$ and $\phi_{2}$ may be accurately approximated as follows using the first two terms of a Taylor series

$$
\begin{gathered}
\phi_{1}\left(\tau_{0}\right)=-0.5+0.216 \tau_{0}+O\left(\tau_{0}{ }^{2}\right) \\
\phi_{2}\left(\tau_{0}\right)=-0.5 \tau_{0}+O\left(\tau_{0}{ }^{2}\right)
\end{gathered}
$$

Substituting these expansions into Eq. (5.6), $\tau_{0}$ is found to equal

$$
\tau_{0}=\frac{0.5 Q_{1}-Q_{4}}{0.216 Q_{1}-0.5 Q_{2}+Q_{3}}+\ldots
$$

From Figure 5.2, the limits of integration for $C_{W+}$ and $C_{W-}$ are identified, which allows the two terms to be written as

$$
\begin{aligned}
C_{W+} & =\int_{0}^{\tau_{0}} C_{P}(\tau) d \tau \\
& =\frac{\Delta \alpha^{2}}{\tau^{* 2}}\left[Q_{1} \Phi_{1}\left(\tau_{0}\right)+Q_{2} \Phi_{2}\left(\tau_{0}\right)+Q_{3} \frac{\tau_{0}{ }^{2}}{2}+Q_{4} \tau_{0}+Q_{5}\right]
\end{aligned}
$$




$$
\begin{aligned}
C_{W-} & =\left|\int_{\tau_{0}}^{\tau^{*}} C_{P}(\tau) d \tau\right| \\
& =-\frac{\Delta \alpha^{2}}{\tau^{* 2}}\left\{Q_{1}\left[\Phi_{1}\left(\tau^{*}\right)-\Phi_{1}\left(\tau_{0}\right)\right]+Q_{2}\left[\Phi_{2}\left(\tau^{*}\right)-\Phi_{2}\left(\tau_{0}\right)\right]+Q_{3} \frac{\tau^{* 2}-\tau_{0}^{2}}{2}+Q_{4}\left[\tau^{*}-\tau_{0}\right]-Q_{5}\right\}
\end{aligned}
$$

where

$$
\begin{gathered}
\Phi_{1}(\tau)=\int \phi_{1}(\tau) d \tau=-2.37152+0.55833 e^{-0.6 \tau}+1.81319 e^{-0.091 \tau} \\
\Phi_{2}(\tau)=\int \phi_{2}(\tau) d \tau=20.8755-2.37152 \tau-0.93055 e^{-0.6 \tau}-19.945 e^{-0.091 \tau}
\end{gathered}
$$

Applying the approximations of Eqs. (5.7 - 5.9), the expression for $C_{W^{+}}$from Eq. (5.10) simplifies to the following

$$
C_{W+}=\frac{\Delta \alpha^{2}}{\tau^{2}}\left[-\frac{1}{2} \frac{\left(0.5 Q_{1}-Q_{4}\right)^{2}}{\left(0.216 Q_{1}+0.5 Q_{3}\right)}+Q_{5}\right]
$$

where the Taylor Series approximations of $\Phi_{1}\left(\tau_{0}\right)$ and $\Phi_{2}\left(\tau_{0}\right)$ are used. Similarly, the approximate equation for $C_{W \text { - }}$ is written as

$$
C_{W-}=-\frac{\Delta \alpha^{2}}{\tau^{*}}\left\{Q_{1} \Phi_{1}\left(\tau^{*}\right)+Q_{2} \Phi_{2}\left(\tau^{*}\right)+Q_{3} \frac{\tau^{* 2}}{2}+Q_{4} \tau^{*}-Q_{5}+\frac{1}{2} \frac{\left(0.5 Q_{1}-Q_{4}\right)^{2}}{\left(0.216 Q_{1}+0.5 Q_{3}\right)}\right\}
$$

where Eq. (5.12) and (5.13) are used for $\Phi_{I}\left(\tau^{*}\right)$ and $\Phi_{2}\left(\tau^{*}\right)$. These equations are valid for values of $x_{a} / c>0.45$ and for $\tau^{*}>0.1$. For values of $\tau^{*}<0.1, \tau_{0}$ is greater than $\tau^{*}$ so that the limits of integration in Eqs. (5.10) and (5.11) are no longer valid. The usefulness of these equations is that they accurately predict the value of $x_{a}$ for the minimum

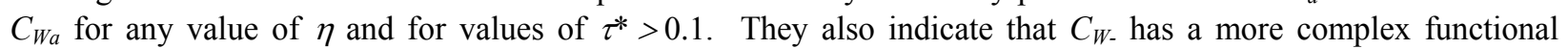
dependence on $\tau^{*}$ than does $C_{W^{+}}$. Figure 5.3 presents the exact values of $C_{W^{+}}$and $C_{W-}$, which were obtained by computing $\tau_{0}$ and specifying the limits of integration for each case. The results of Eq. (5.14) for $C_{W^{+}}$are shown as a dashed line for each case. It is seen that the results of Eq. (5.14) are indistinguishable from the exact result for $x_{a} / c>$ 0.45 and become invalid as $x_{a} / c$ approaches 0.25 . The result of Eq. (5.15) is not shown in Figure 5.3, although it can be shown to be accurate for the same values of $x_{a}$ as Eq. (5.14). This figure shows that $C_{W+}$ and $C_{W-}$ converge to the limit of $\tau^{*}=$ infinity, which represents the results of steady airfoil theory. It also shows that, as expected from steady airfoil theory, $C_{W^{+}}$is largest for $x_{a} / c<0.25$ and $C_{W^{-}}$is largest for $x_{a} / c>0.25$. The pitching axis for minimum $C_{W^{+}}$is found exactly from Eq. (5.14) to equal 0.572, which is independent of $\tau^{*}$. Figure 5.3 verifies that this minimum is located within the range of $x_{a}$ values where Eq. (5.14) is valid. From the $C_{W}$ - plot in Figure 5.3 it is deduced that as $\eta$ becomes nonzero and positive, the minimum $C_{W a}$ pitching axis shifts towards the leading edge. Similarly, as $\eta$ becomes negative, the optimal axis shifts to the trailing edge. 

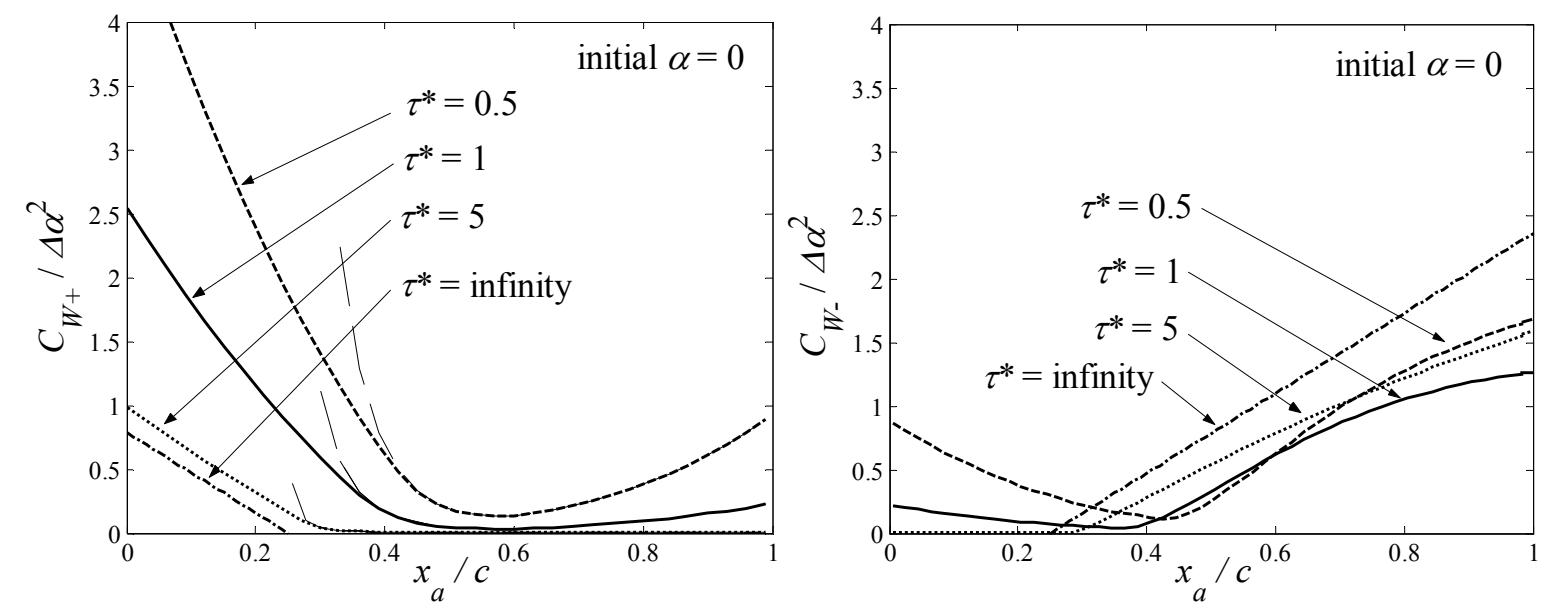

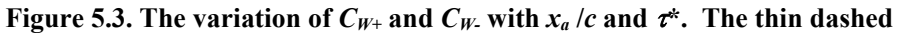
lines in the $C_{W+}$ plot represent the result of Eq. (5.14).

The cases shown in Figure 5.2 and discussed previously specified that the initial $\alpha$, and therefore the initial lift, was zero. The effect of an initial lift will now be presented. From Eq. (4.7) it is seen that an initial angle of attack $\left(\alpha_{0}\right)$ only influences $C_{P}$ through the last term, which contains $Q_{3}$. Dividing this equation by $\Delta \alpha^{2}$ allows $C_{P}$ to be written as follows

$$
\frac{C_{P}}{\Delta \alpha^{2}}=\frac{1}{\tau^{*}}\left[Q_{1} \phi_{1}(\tau)+Q_{2} \phi_{2}(\tau)+Q_{3} \tau+Q_{4}+\left(\delta(\tau)-\delta\left(\tau-\tau^{*}\right)\right) Q_{5}\right]+\frac{k}{\tau^{*}} Q_{3}
$$

where

$$
k=\frac{\alpha_{0}}{\Delta \alpha}
$$

The value $k$ represents the initial lift divided by the change in steady state lift. Recognizing the term $k$ in Eq. (5.16) is useful because it indicates that the normalized power coefficient $\left(C_{P} / \Delta \alpha^{2}\right)$ is dependent only on the ratio of $\alpha_{0}$ and $\Delta \alpha$, and not each term independently. The presence of $k$ significantly complicates the problem of analytically

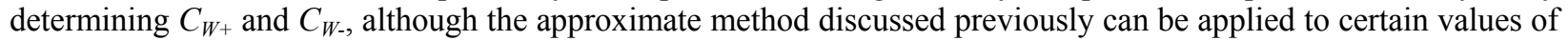
$k$. The main effect of the initial lift is to vertically displace the $C_{P}$ curves, such as those shown in Figure 5.2. This significantly changes $\tau_{0}$ and therefore alters the allocation of $C_{W}$ into $C_{W^{+}}$and $C_{W_{-}}$terms.

To gain some insight into the effect of $k$ on $C_{W a}$, the limiting cases of $\tau^{*}$ approaching zero and infinity will be examined. For $\tau^{*}$ approaching zero, the region of integration for $C_{W^{+}}$is $0 \leq \tau<\tau^{*}$, and the $C_{W-}$ component comes completely from the Dirac delta function at $\tau^{*}$. From Eq. (5.16), the integration for $C_{W a}$, with $\tau^{*}$ approaching zero, results in

$$
\frac{C_{W_{a}}}{\Delta \alpha^{2}}=\frac{1}{\tau^{*}}\left[-\frac{1}{2} Q_{1}+Q_{4}\right]+\frac{(1+\eta) Q_{5}}{\tau^{* 2}}+O(1)
$$

which is independent of $k$. For $\eta>-1$, the $Q_{5}$ term is dominant. Thus, from Eq. (5.5) the pitching axis for minimum $C_{W a}$ is at the half chord. For $\eta=-1$, only the bracketed term remains in Eq. (5.18). Substituting Eqs. (5.2) and (5.4) into (5.18) and setting the derivative with respect to $x_{a}$ equal to zero, the pitching axis for minimum $C_{W a}$ is found to be located at $x_{a} / c=3 / 4$.

For $\tau^{*}$ approaching infinity, the region of integration for $C_{W+}$ and $C_{W-}$ depends upon $k$ and $x_{a}$. This is seen by writing Eq. (5.16) in terms of its lowest order components for large values of $\tau^{*}$. To determine the lowest order components, it is necessary to define $\tau$ as

$$
\tau=\bar{\tau} \tau *
$$

where $0<\bar{\tau}<1$. Substituting this into Eq. (5.16), the lowest order equation for $C_{P}$ is written as follows 


$$
\frac{C_{P}}{\Delta \alpha^{2}}=\frac{Q_{3}}{\tau^{*}}(\bar{\tau}+k)+O\left(\frac{\ln \tau^{*}}{\tau^{*}}\right)
$$

Note that, as pointed out by Lomax ${ }^{17}$, the asymptotic limits of $\phi_{1}$ and $\phi_{2}$ obtained from Eqs. (4.5) and (4.6) are incorrect. Therefore, the approximate Wagner function suggested by Garrick ${ }^{40}$ was used instead for obtaining Eq. (5.20). As expected, Eq. (5.20) represents the steady thin airfoil theory result. Equation (5.20) shows that, if $k$ is less than - 1 or greater than zero, the lowest order component of $C_{W a}$ is composed entirely of either $C_{W+}$ or $C_{W-\text {. For }}$ these values of $k, C_{W a}$, is written from Eq. (5.20) and (5.3) as follows

$$
\begin{gathered}
F\left(x_{a}, k\right)=\frac{\pi}{2}\left[\left(\frac{1}{2}+k\right)\left(1-4 x_{a}\right)\right]+O\left(\frac{\ln \tau^{*}}{\tau^{*}}\right) \\
\text { if } F \geq 0, \frac{C_{W_{a}}}{\Delta \alpha^{2}}=F\left(x_{a}, k\right) \\
\text { if } F<0, \frac{C_{W_{a}}}{\Delta \alpha^{2}}=\eta\left|F\left(x_{a}, k\right)\right|
\end{gathered}
$$

For values of $k$ between -1 and zero, $\tau_{0}$ is determined by setting Eq. (5.20) equal to zero. This value of $\tau_{0}$ is then used as a limit of integration for $C_{W a}$, which from Eqs. (5.20) and (5.3) results in

$$
\begin{aligned}
& \text { if } x_{a}<1 / 4, \frac{C_{W_{a}}}{\Delta \alpha^{2}}=\frac{\pi}{2}\left(\frac{1}{2}+k+\frac{k^{2}}{2}\right)\left(1-4 x_{a}\right)+\eta \pi k^{2}\left(\frac{1}{4}-x_{a}\right)+O\left(\frac{\ln \tau^{*}}{\tau^{*}}\right) \\
& \text { if } x_{a} \geq 1 / 4, \frac{C_{W_{a}}}{\Delta \alpha^{2}}=-\pi k^{2}\left(\frac{1}{4}-x_{a}\right)-\eta \frac{\pi}{2}\left(\frac{1}{2}+k+\frac{k^{2}}{2}\right)\left(1-4 x_{a}\right)+O\left(\frac{\ln \tau^{*}}{\tau^{*}}\right)
\end{aligned}
$$

Table 5.1 presents the pitching axes for minimum $C_{W a}$ obtained from Eqs. (5.21) and (5.22) with the constraint that the axes remain within the chord. These results are intuitive from the elementary nature of a steady thin airfoil at an angle of attack.

Table 5.1. The minimum $C_{W a}$ pitching axes as $\tau^{*}$ approaches infinity

Table 5.1. The minimum $C_{W a}$ pitching axes as $\tau^{*}$ approaches infinity
\begin{tabular}{|c|c|c|c|c|}
\hline & $k<-1$ & $-1<k<-1 / 2$ & $-1 / 2<k<0$ & $k>0$ \\
\hline$\eta=1$ & $x_{a} / c=1 / 4$ & $x_{a} / c=1 / 4$ & $x_{a} / c=1 / 4$ & $x_{a} / c=1 / 4$ \\
\hline$\eta=0$ & $0<x_{a} / c<1 / 4$ & $x_{a} / c=1 / 4$ & $x_{a} / c=1 / 4$ & $1 / 4<x_{a} / c<1$ \\
\hline$\eta=-1$ & $x_{a} / c=0$ & $x_{a} / c=0$ & $x_{a} / c=1$ & $x_{a} / c=1$ \\
\hline
\end{tabular}

The limiting cases of $\tau^{*}$ discussed above allowed $C_{W a}$ to be obtained analytically, which allowed the optimal pitching axes to be determined analytically. For the $k \geq 0$ cases, the approximate approach presented in Eqs. (5.9 5.15), accounting for the $k$ term in Eq. (5.16), is valid for a wide range of $\tau^{*}$ values. Where this approach is not valid, the integration for $C_{W a}$ is performed numerically from Eqs. (4.13), (4.14), and (5.16). Using a combination of analytic and numerical approaches, the minimum $C_{W a}$ pitching axes were obtained for $\eta=0,1$, and -1. Figure 5.5 shows the variation of the optimal pitching axis with $\tau^{*}$ for the $\eta=0$ case for various $k$ values. As determined previously, the axes are shown to approach $x_{a} / c=0.5$ as $\tau^{*}$ approaches zero. It is seen in this figure that as $k$ becomes large and positive, the optimal axis is located at $x_{a} / c=0.5$ for most $\tau^{*}$ values. This is a result of $C_{W^{+}}$being composed of only the initial impulse, which is smallest for the mid-chord axis. For negative $k$ values, the optimal axis moves toward the leading edge as $\tau^{*}$ increases. Figure 5.5 shows the variation of the optimal pitching axis with $\tau^{*}$ for the $\eta=1$ case and various $k$ values. It is interesting to note that for this case, as was determined previously, the optimal axis at both $\tau^{*}$ equal to zero and infinity is independent of $k$. This explains the increased similarity between the optimal axes curves for various $k$ values in Figure 5.5 when compared to Figure 5.4. For airfoils that must complete a cycle, meaning they produce a change in lift (positive $k$ ) and then later produce a negative change in lift to return to their initial state (negative $k$ ), the similarity in the optimal axes for negative and positive $k$ values is advantageous. This is because a smaller compromise must be made, assuming the pitching axis remains fixed, 
when choosing the optimal pitching axis for the complete motion. For the majority of negative and positive combinations of $k$, the optimal axis for the combination is located between the two independent optimal points for a given $\tau^{*}$. Thus, Figures 5.4 and 5.5 are very general and applicable to many practical cases. Figure 5.6 presents the variation of the optimal pitching axis with $\tau^{*}$ for the $\eta=-1$ case. It is seen that the difference between positive and negative $k$ values is very large compared to Figures 5.4 and 5.5. The result of increasing $k$ in Figure 5.6 is seen to be a decrease in the value of $\tau^{*}$ at which the optimal axis is the same as those shown in Table 5.1 for the $\tau^{*}$ equal to infinity case. The same conclusion can be stated from Figures 5.5. A similar result was reported by Yates ${ }^{41}$ for the minimum energy pitching axes of an oscillating flat plate intended to produce thrust.

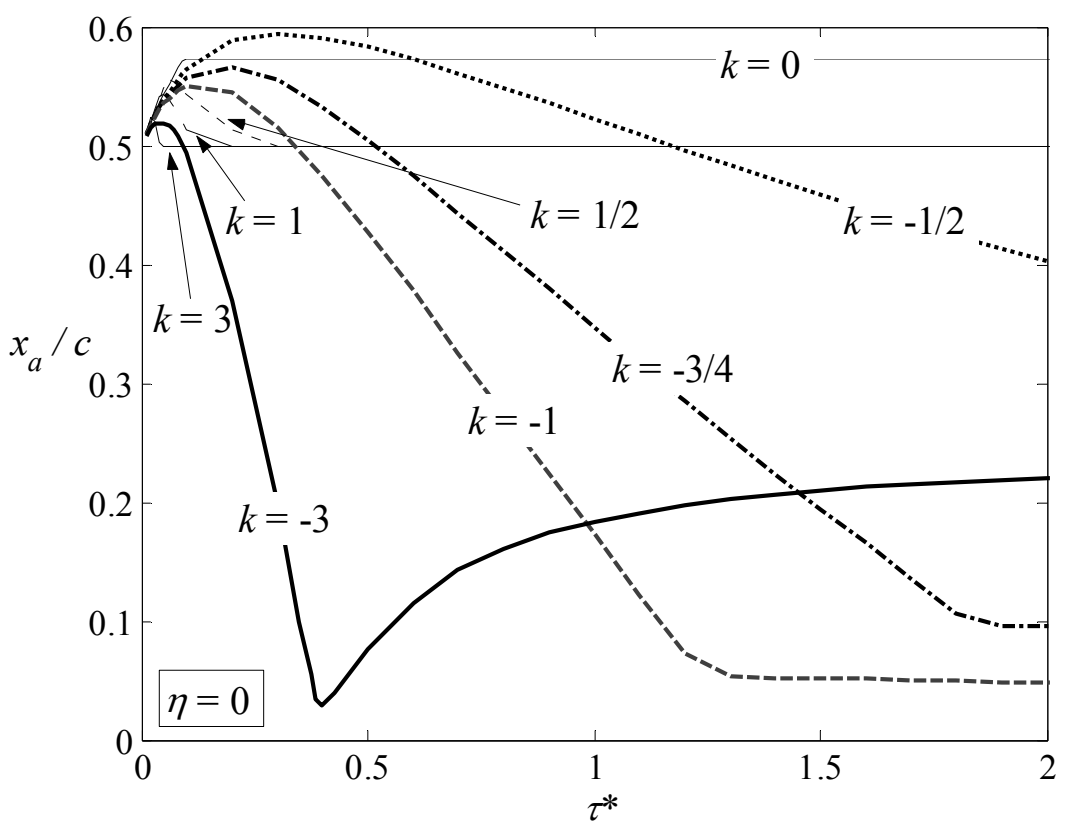

Figure 5.4. The $\eta=0$ case for the variation of the minimum $C_{W a}$ pitching axes with $\tau^{*}$ for various values of $\boldsymbol{k}$.

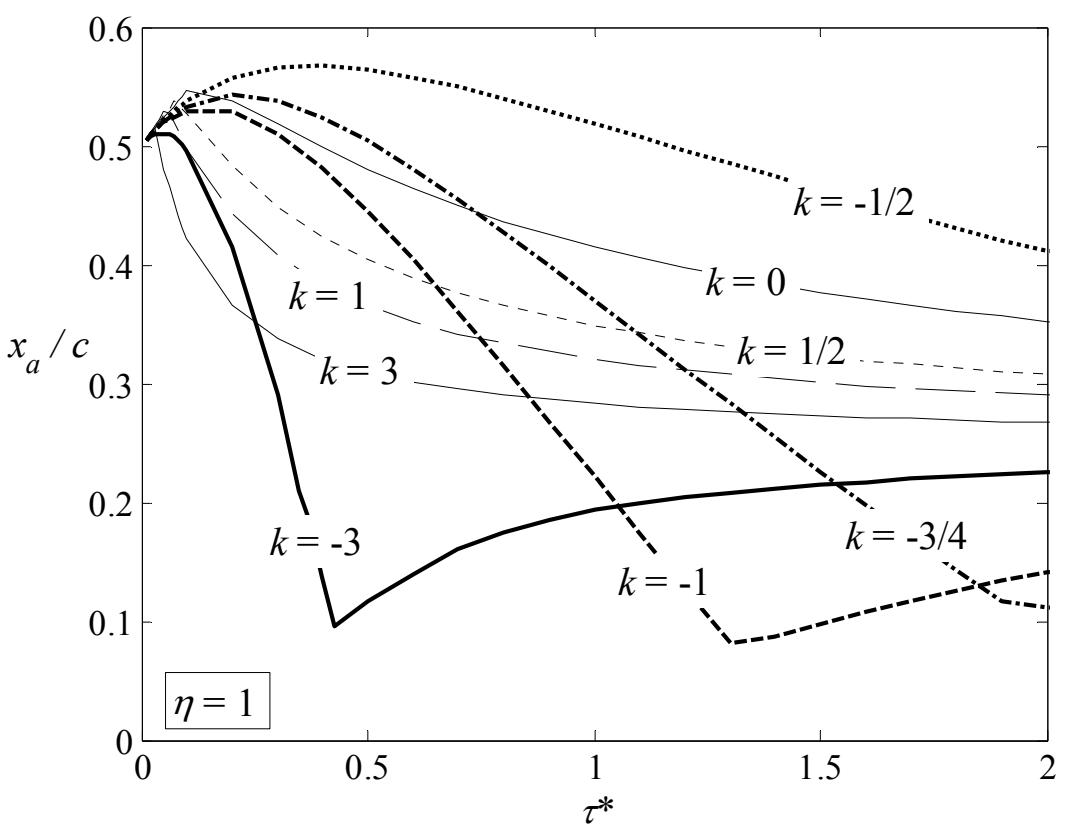

Figure 5.5. The $\eta=1$ case for the variation of the minimum $C_{W a}$ pitching axes with $\tau^{*}$ for various values of $k$. 


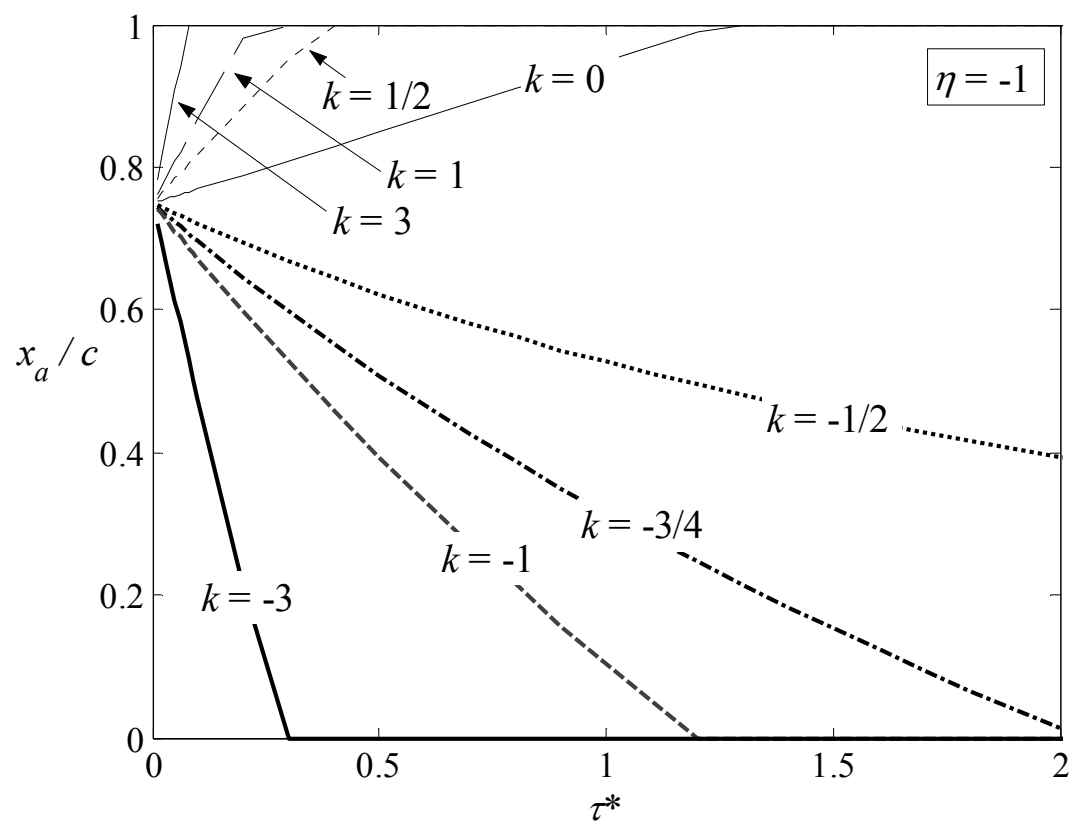

Figure 5.6. The $\eta=-1$ case for the variation of the minimum $C_{W a}$ pitching axes with $\tau^{*}$ for various values of $k$.

\section{Application to Various Control Surface Configurations}

This section describes the affect of various control surface shapes on the $C_{W a}$ required for a given change in lift. The first two cases to be considered are shown in Figures 6.1 and 6.2. A conventional hinged flap is shown in Figure 6.1. The conformal control surface, shown in Figure 6.2, is a quadratic segment defined to have zero slope at $x_{b}$. The magnitude of the flap deflection $(\beta)$ is defined in both cases as the angle at the trailing edge. The ramp input of $\beta$, defined in Eq. (4.1), will be used for this analysis. From the shape functions ( $\psi$ ), which are shown for each case in Figures 6.1 and 6.2, the components of $\Delta C_{p}$ in Eq. (4.3) may be determined analytically from the equations of Section III. The resulting equations are relatively complex, and it is therefore convenient to perform the integrations required for the $Q$-terms defined in Eqs. $(4.8-4.12)$ numerically.

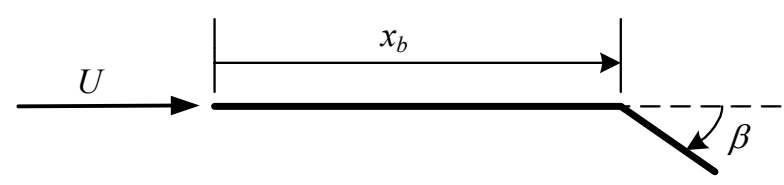

$$
\begin{gathered}
0 \leq x<x_{b}, \\
\psi(x)=0 \\
x_{b} \leq x \leq c, \\
\psi(x)=-x+x_{b}
\end{gathered}
$$

Figure 6.1. The camberline geometry for a conventional flap

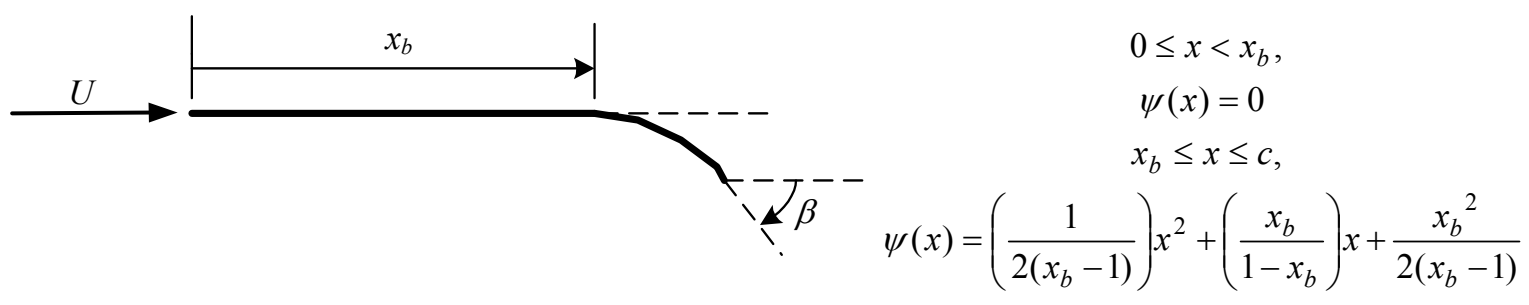

Figure 6.2. The camberline geometry for a conformal flap 
Note that in the previous case of the pitching flat plate, the $\Delta C_{L}$ produced by a $\Delta \alpha$ was independent of the pitching axis. This meant that the $C_{W a}$ required for a given lift could be represented by $C_{W a} / \Delta \alpha^{2}$. For comparing various control surface configurations, it is convenient to instead normalize $C_{P}$ and $C_{W a}$ by the $\Delta C_{L}{ }^{2}$. From Eq. (4.7), the normalized equation for $C_{P}$ can then be written as

$$
\frac{C_{P}}{\Delta C_{L}^{2}}=\frac{1}{\tau^{* 2} K_{0, s}^{2}}\left[Q_{1} \phi_{1}(\tau)+Q_{2} \phi_{2}(\tau)+Q_{3} \tau+Q_{4}+\left(\delta(\tau)-\delta\left(\tau-\tau^{*}\right)\right) Q_{5}\right]+\frac{k}{\tau^{*} K_{0, s}} Q_{3}
$$

where

$$
k=\frac{\bar{\beta}_{0}}{\Delta \bar{\beta}}=\frac{C_{L, \text { initial }}}{\Delta C_{L}}
$$

Recall that the quantity $\Delta C_{L}$ refers to the change in steady state lift, which from Eq. (3.7) is written as

$$
\Delta C_{L}=K_{0, s} \Delta \bar{\beta}
$$

Considering the conventional and conformal flap configurations, if $k$ is greater than zero, then $C_{P}$ remains positive throughout the ramp input of $\beta$. Therefore, $C_{W^{+}}$is obtained by integrating Eq. (6.1) from $\tau=0$ to $\tau^{*}$ and $C_{W_{-}}$is obtained from Eq. (4.16). For small negative values of $k, C_{P}$ changes from positive to negative and therefore $\tau_{0}$ must be determined. For these cases, the process described with Eqs. $(5.9-5.15)$ may be used. For large negative $k$ values, $C_{P}$ remains negative throughout the ramp input of $\beta$. Therefore, $C_{W-}$ is obtained by integrating Eq. (6.1) from $\tau=0$ to $\tau^{*}$ and $C_{W^{+}}$is obtained from Eq. (4.16).

It is desired to compare the values of $C_{W a}$ resulting from the conventional and conformal flap configurations defined in Figures 6.1 and 6.2. The first case to be considered, shown in Figure 6.3, compares the $C_{W a}$ required for a given $\Delta C_{L}, x_{b}$, and $\tau^{*}$ while varying $k$. It is seen that the $C_{W a}$ required by the conformal flap is less than that required by the conventional flap for any $k$ when $\eta=0$. For the $\eta=1$ case, there is a small range of $k$ values where $C_{W a}$ is slightly less for the conventional flap. Overall though, the conformal flap requires less $C_{W a}$ than the conventional flap.

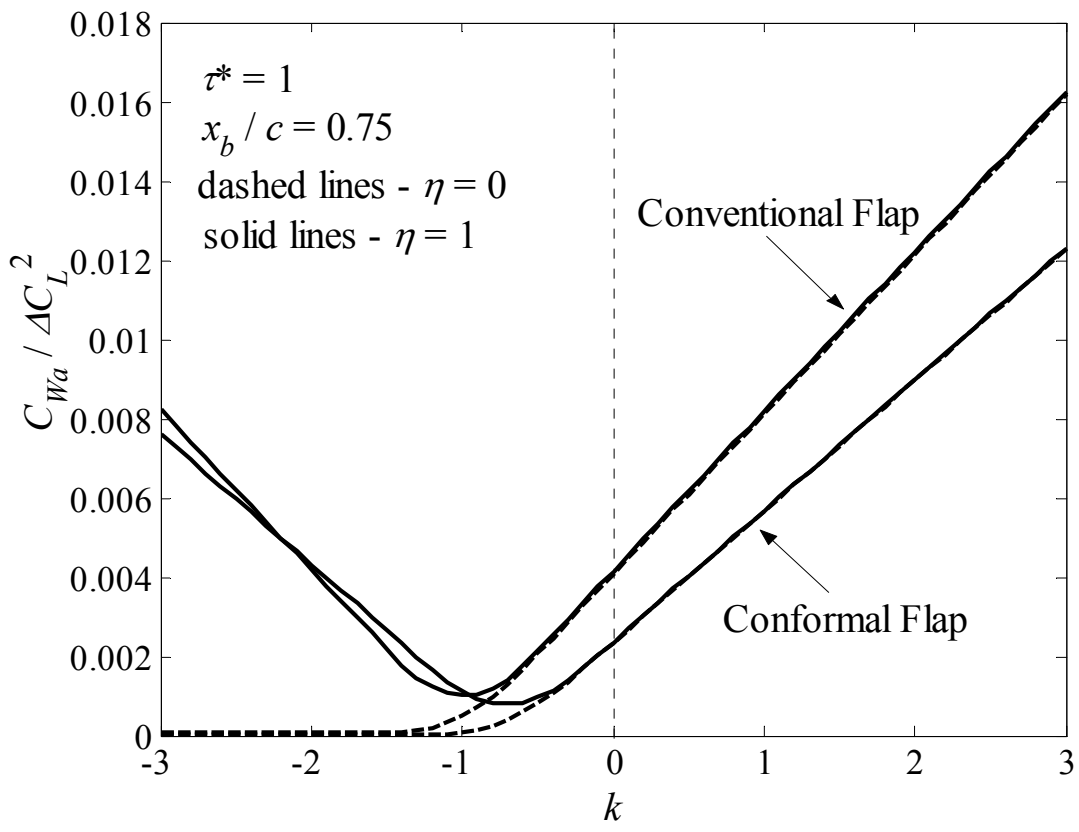

Figure 6.3. A comparison of the $C_{W a}$ required for a conformal or conventional flap.

The reason for the smaller $C_{W a}$ for the conformal flap is that it requires less overall camberline deformation for a given change in lift than does the conventional flap. Figure 6.4 illustrates this result along with the corresponding 
load distribution at $\tau=1 / 2$. It is seen that the angle of deflection at the trailing edge of the conformal flap is larger than that for the conventional flap for a given change in lift, but the overall $\Delta z$ of the camberline is less for the conformal flap. The load distribution for the conventional flap is centered more towards the hinge-line than for the conformal flap, which is favorable for the conventional flap. Nevertheless, the larger $\Delta z$ overshadows the favorable load distribution for the conventional flap. It should be mentioned that the shape of the load distributions shown in Figure 6.4 apply only at $\tau=1 / 2$. As shown in Eq. (4.3), the load distribution does not simply scale linearly with the ramp input of $\beta$.
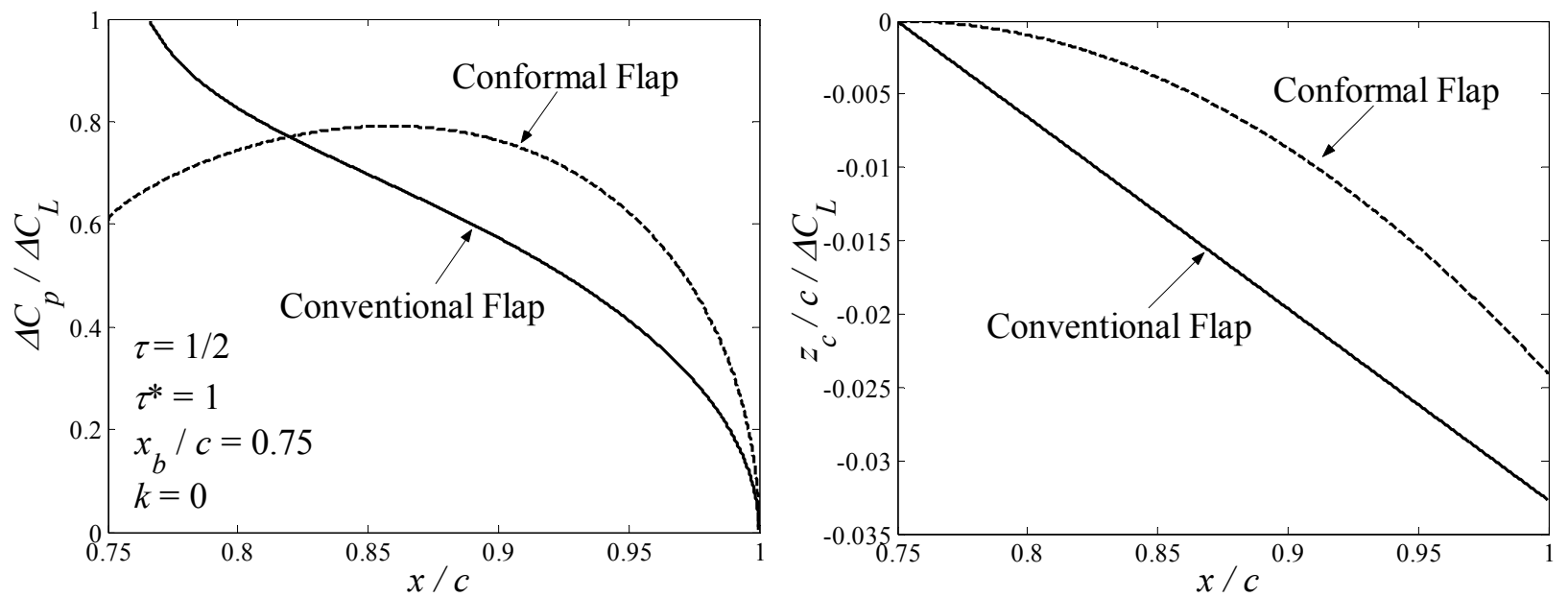

Figure 6.4. The load distribution over the flap and the corresponding shape of the flap deflections

Figure 6.5 shows how $C_{W a}$ varies with $\tau^{*}$ and $x_{b}$ for the conformal and conventional flap. It is seen that the conformal flap requires less $C_{W a}$ for every case. It is also apparent that the benefit of the conformal flap becomes larger as $\tau^{*}$ decreases. Hence, the conformal flap is ideal in situations where rapid changes in lift are required. The values of $C_{W a}$ in the limit as $\tau^{*}$ goes to infinity are shown in Figure 6.5. These values, which can be obtained from steady thin airfoil theory, show that $C_{W a}$ is $18 \%$ less for the conformal flap in the steady limit. The considerable difference between the steady and unsteady values in Figure 6.5 indicates the importance of including the unsteady aerodynamic terms in this analysis. It should be mentioned that the values of $C_{W a}$ for a given change in quarter chord pitching moment $\left(C_{M}\right)$, produce results similar to those in Figure 6.5. In particular, the value of $C_{W a} / C_{M}{ }^{2}$ decreases continuously as $x_{b}$ varies from midchord to the trailing edge. This is true even though the flap deflection required to produce a pitching moment has a minimum at $x_{b} / c=0.75$ for the conventional case.
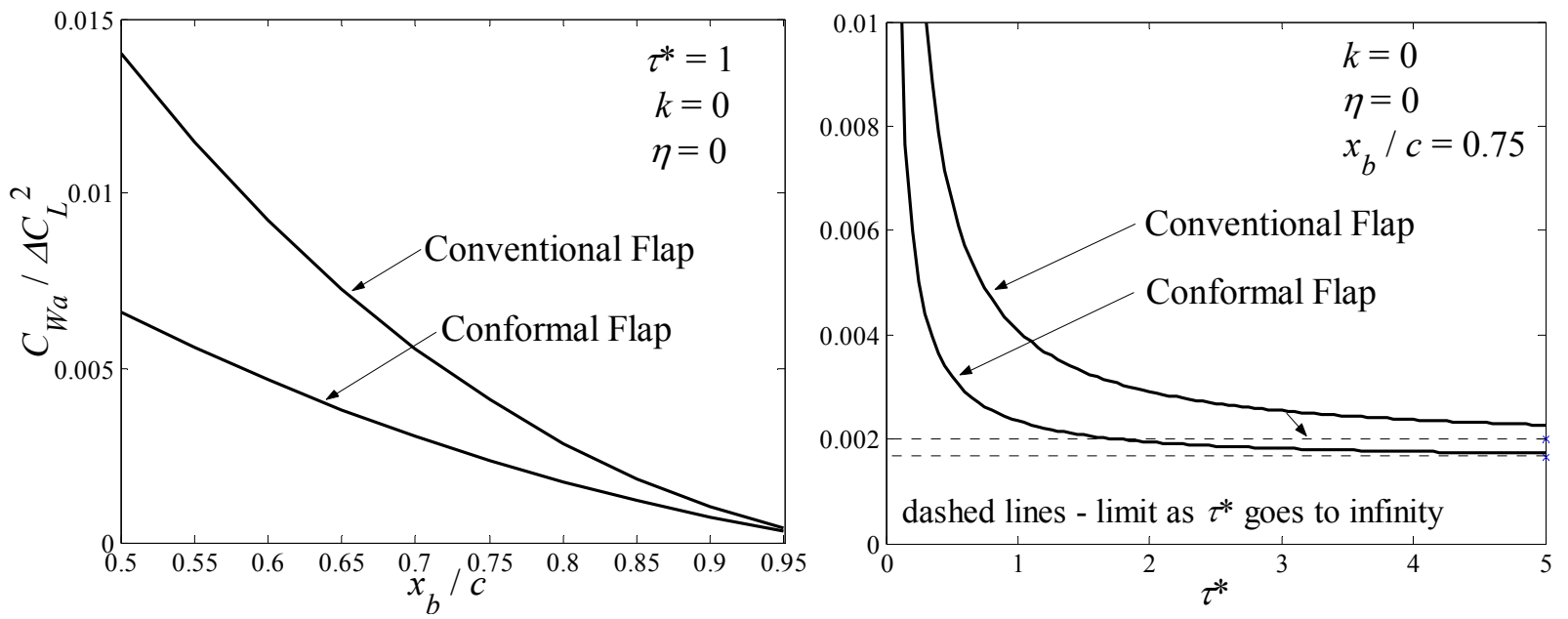

Figure 6.5. The effect of flap size and $\tau^{*}$ on the $C_{W a}$ required for the conformal or conventional flap.

The next cases to be considered are the variable camber configurations shown in Figures 6.6 and 6.7, which are defined as NACA 4-digit camberlines with time-dependent magnitudes of maximum camber. Configuration A, 
shown in Figure 6.6, is defined so that the leading and trailing edges remain on the $x$-axis as the camber changes. Configuration B, shown in Figure 6.7, is defined so that the location of maximum camber $\left(x_{b}\right)$ remains on the $x$-axis as the camber changes. Figure 3.1 shows that in steady thin airfoil theory, these two configurations produce the same aerodynamic forces. But, the addition of the aerodynamic damping component, shown in Figure 3.2, makes the unsteady thin airfoil results different between the two cases. In considering the actuator energy for each case, it is assumed that each configuration is actuated with a single actuator. This implies that some type of linkage system is used to produce the desired camberline shape. Also, as has been done throughout this paper, only the aerodynamic forces are considered for the actuator energy. It is recognized that this is a big assumption for these variable camber configurations, but nonetheless, we feel that the present analysis provides significant insight into the actuation properties of a variable camber airfoil.

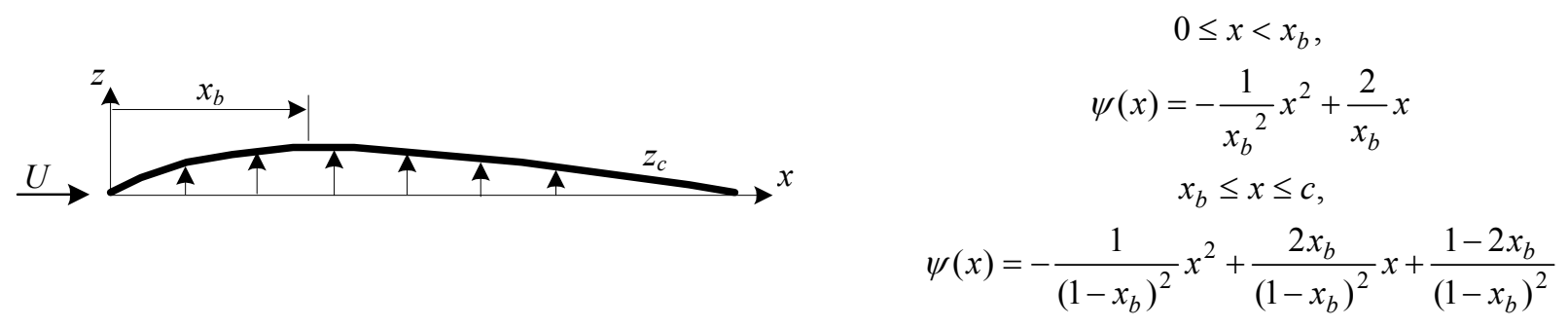

Figure 6.6. The camberline geometry for a variable camber airfoil with the leading and trailing edges fixed to the $\mathbf{x}$-axis (Configuration $\mathbf{A})$.

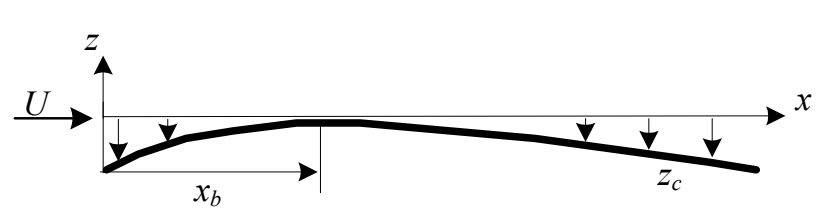

$$
\begin{gathered}
0 \leq x<x_{b}, \\
\psi(x)=-\frac{1}{x_{b}{ }^{2}} x^{2}+\frac{2}{x_{b}} x-1 \\
x_{b} \leq x \leq c, \\
\psi(x)=-\frac{1}{\left(1-x_{b}\right)^{2}} x^{2}+\frac{2 x_{b}}{\left(1-x_{b}\right)^{2}} x-\frac{x_{b}{ }^{2}}{\left(1-x_{b}\right)^{2}}
\end{gathered}
$$

Figure 6.7. The camberline geometry for a variable camber airfoil with $x_{b}$ fixed to the $x$-axis (Configuration B).

The dependence of $C_{W a}$ on $k$ and $x_{b} / c$ is shown in Figure 6.8 for both configurations and $\eta=0$. It is seen that configuration B requires significant $C_{w a}$ for positive $k$ cases while configuration A requires very little for these cases. This result is explained by recognizing that the camberline motion for configuration $\mathrm{B}$ is downwards for a positive change in lift, which must therefore move against the upward acting lift forces. On the other hand, the camberline motion for configuration $\mathrm{A}$ is upwards and is therefore not resisted by the aerodynamic forces. For negative $k$ values, the situation reverses and this configuration requires significant $C_{W a}$. Figure 6.8 shows that configuration B requires less $C_{W a}$ for a given positive $k$ than configuration A requires for a negative $k$ of the same magnitude. This means that if the airfoil is intended to produce an equal number of positive changes in lift as negative changes in lift, then configuration B is favorable from an energy standpoint. The second plot in Figure 6.8 shows that this conclusion is true for any location of maximum camber $\left(x_{b}\right)$. It is also seen that as $x_{b}$ moves closer to the leading edge, configuration $\mathrm{B}$ becomes even more favorable. 

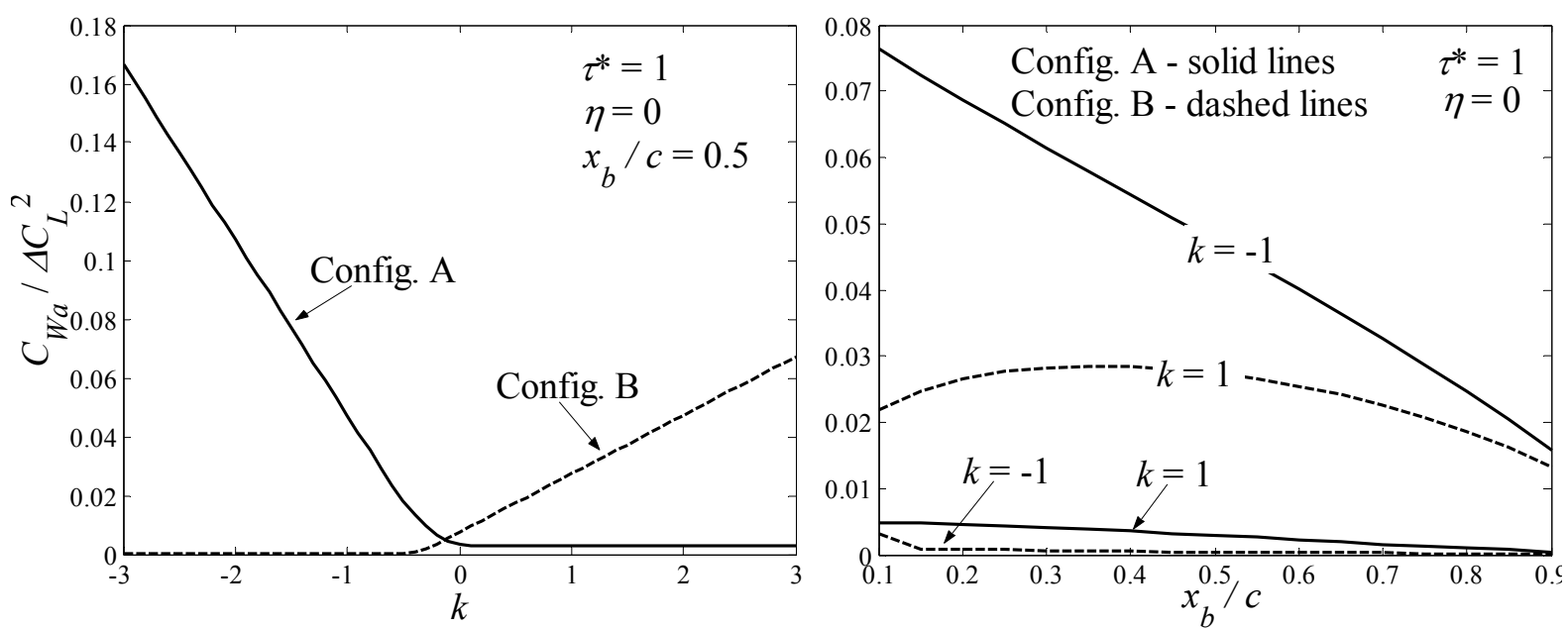

Figure 6.8. The effect of $k$ and $x_{b} / c$ on the $C_{W a}$ required for configuration $A$ and B.

The load distribution and corresponding camberline shape at $\tau=1 / 2$ are shown in Figure 6.9. This figure illustrates the point made previously that the camberline motion for configuration B is resisted by the aerodynamic forces for $k$ greater than or equal to zero. Note that the difference between the load distributions shown in this figure comes from the $K_{0, d}$ and $\bar{A}_{0, d}$ terms in Eq. (4.3). This figure makes clear the reasons why configuration B requires less $C_{W a}$ (when considering the entire range of $k$ values) than configuration A. The first reason is that configuration B simply requires less overall camberline deflection than configuration $\mathrm{A}$. The second reason is that for configuration $\mathrm{A}$, the largest camberline deflections are towards the center of the camberline while for configuration B they are at the leading and trailing edges. Combining this fact with the shape of the load distribution makes clear the advantage of configuration $\mathrm{B}$.
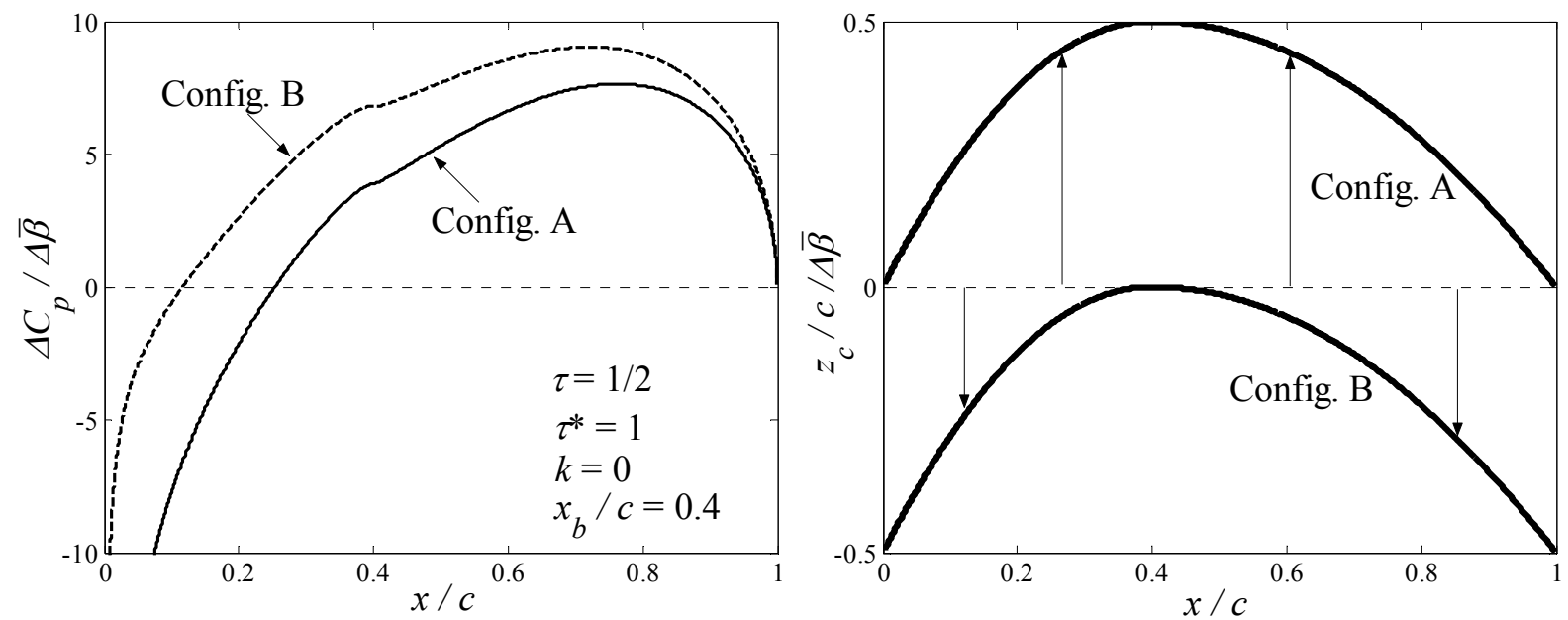

Figure 6.9. Example of the unsteady load distribution and corresponding camberline shape for configuration $A$ and $B$.

\section{Conclusions}

The work required to overcome the aerodynamic forces to produce a change in lift through camberline deformation was shown to depend significantly on the initial lift of the airfoil. This conclusion arises because there is infinite energy in a lifting two-dimensional flow. The power required for a ramp input of arbitrary camberline deformation was shown to depend on five terms, defined as $Q_{1}, Q_{2}, \ldots, Q_{5}$, which depend on the results of unsteady thin airfoil theory. The necessity of using unsteady thin airfoil theory for the study was illustrated. The pitching axis required for a flat plate to produce a change in lift with minimum energy input to the actuator was shown to depend on the energy required by the actuator to produce negative work. Assuming that there is no energy cost 
associated with negative work, the minimum energy pitching axis for an airfoil with zero initial lift is located at $x / c$ equal to 0.572 for a ramp input. For various actuator models, the minimum energy pitching axes were obtained and shown to depend on the rate of the ramp input $\left(\tau^{*}\right)$. A conformal flap was shown to require significantly less energy than a conventional flap to produce a change in lift. This conclusion was shown to be independent of the initial lift, rate of the flap deflection, and flap size. A downward deflecting variable camber configuration (configuration $\mathrm{B}$ ) was shown to require less energy than an upward deflecting configuration (configuration A) if both positive and negative changes in lift are considered. Among the control devices investigated in this paper, the conformal trailing edge flap requires the least energy to overcome the aerodynamic forces for a given change in lift.

\section{Acknowledgments}

This work was performed with the financial support of the Center for Intelligent Material Systems and Structures (CIMSS). This support is gratefully acknowledged.

\section{References}

${ }^{1}$ Stanewsky, E., “Aerodynamic Benefits of Adaptive Wing Technology,” Aerospace Sciences and Technology, Vol. 4, 2000, pp. 439-452.

${ }^{2}$ Stanewsky, E., “Adaptive Wing and Flow Control Technology,” Progress in Aerospace Sciences, Vol. 37, 2001 , pp. $583-667$.

${ }^{3}$ Forster, E., Sanders, B., and Eastep, F., "Synthesis of a Variable Geometry Trailing Edge Control Surface," AIAA Paper 20031717, April 2003.

${ }^{4}$ Forster, E., Sanders, B., and Eastep, F., "Modeling and Sensitivity Analysis of a Variable Geometry Trailing Edge Control Surface," AIAA Paper 2003-1807, April 2003.

${ }^{5}$ Gern, F. H., Inman, D. J., and Kapania, R. K., "Computation of Actuation Power Requirements for Smart Wings with Morphing Airfoils," AIAA Paper 2002-1629, April 2002.

${ }^{6}$ Pettit, G. W., Robertshaw, H. H., Gern, F. H., and Inman, D. J., "A Model to Evaluate the Aerodynamic Energy Requirements of Active Materials in Morphing Wings," 2001 ASME Design Engineering Technical Conference, September 2001.

${ }^{7}$ Prock, B. C., Weisshaar, T. A., and Crossley, W. A., "Morphing Airfoil Shape Change Optimization with Minimum Actuator Energy as an Objective," AIAA Paper 2002-5401, September 2002.

${ }^{8}$ Sanders B., Eastep, F. E., and Forster, E., "Aerodynamic and Aeroelastic Characteristics of Wings with Conformal Control Surfaces for Morphing Aircraft," Journal of Aircraft, Vol. 40, Jan.-Feb. 2003, pp. 94-99.

9 von Karman, T., and Burgers, J. M., "General Aerodynamic Theory - Perfect Fluids," Aerodynamic Theory, W. F. Durand, ed., Vol. II, Julius Springer, 1935, pp. 304-310.

${ }^{10}$ Garrick, I. E., "Propulsion of a Flapping and Oscillating Airfoil," NACA Report No. 567, 1936.

${ }^{11} \mathrm{Wu}, \mathrm{T}$. Y., "Hydromechanics of Swimming Propulsion, Part 1. Swimming of a Two-Dimensional Flexible Plate at Variable Forward Speeds in an Inviscid Fluid," Journal of Fluid Mechanics, Vol. 46, part 2, 1971, pp. 337-355.

${ }^{12}$ Wu, T. Y., "Swimming of a Waving Plate," Journal of Fluid Mechanics, Vol. 10, 1961, pp. 321-344.

${ }^{13} \mathrm{Wu}, \mathrm{T}$. Y., "Extraction of Flow Energy by a Wing Oscillating in Waves," Journal of Ship Research, March, 1972, pp. 66-78.

${ }^{14}$ Send, W., "The Mean Power of Forces and Moments in Unsteady Aerodynamics," ZAMM, Vol. 72, 1992, pp. $113-132$.

${ }^{15}$ Patil, M. J., "From Fluttering Wings to Flapping Flight: The Energy Connection," Journal of Aircraft, Vol. 40, No. 2, MarchApril, 2003.

${ }^{16}$ Jones, K. D., and Platzer, M. F., "Numerical Computation of Flapping-Wing Propulsion and Power Extraction,” AIAA paper 97-0826, Jan. 1997.

${ }^{17}$ McKinney, W., DeLaurier, J., “The Wingmill: An Oscillating-Wing Windmill,” Journal of Energy, Vol. 5, No. 2, March-April, 1981, pp. 109-115.

${ }^{18} \mathrm{Wu}, \mathrm{T}$. Y., "Hydromechanics of Swimming Propulsion, Part 2. Some Optimum Shape Problems," Journal of Fluid Mechanics, Vol. 46, part 3, 1971, pp. 521-544.

${ }^{19}$ Jones, R. T., Wing Theory, Princeton University Press, 1990, pp. 59-65.

${ }^{20}$ Lomax, H., "Indicial Aerodynamics," in AGARD Manual on Aeroelasticity, Part II, Chapter 7, June, 1960.

${ }^{21}$ Fleischer, H., Manual of Pneumatic Systems Optimization, McGraw Hill, Inc., 1995.

${ }^{22}$ Green, W. L., Aircraft Hydraulic Systems, John Wiley and Sons, 1985.

${ }^{23}$ Weis-Fogh, T., "Quick Estimates of Flight Fitness in Hovering Animals, Including Novel Mechanisms of Lift Production," Journal of Experimental Biology, Vol. 59, 1973, pp. 169-230.

${ }^{24}$ Abbot, B. C., Bigland, B., and Ritchie, J. M., "The Physiological Cost of Negative Work, Journal of Physiology, Vol. 117, 1952, pp. 380-390.

${ }^{25}$ Postel, E. E., and Leppert, E. L., "Theoretical Pressure Distribution for a Thin Airfoil Oscillating in Incompressible Flow," Journal of the Aeronautical Sciences, Vol. 15, No. 8, August 1948, pp.486-492. 


\footnotetext{
${ }^{26}$ Spielburg, I. N., "The Two-Dimensional Incompressible Aerodynamic Coefficients for Oscillatory Changes in Airfoil Camber," Journal of the Aeronautical Sciences, Vol. 20, No. 6, June 1953, pp.432-433.

${ }^{27}$ Mateescu, D., and Abdo, M., "Unsteady Aerodynamic Solutions for Oscillating Airfoils," AIAA paper 2003-227, 2003.

${ }^{28}$ Han, C., Johnston, C. O., Mason. W. H., "Unsteady Thin Airfoil Theory for Deforming Airfoils," AIAA Journal, (to be published).

${ }^{29}$ von Karman, T., and Sears, W. R., “Airfoil Theory for Non-Uniform Motion,” Journal of the Aeronautical Sciences, Vol. 5, No. 10, August, 1938, pp. 378-390.

${ }^{30}$ Abzug, M. J., "Estimation of the Lift and Moment Parameters of Leading-Edge Flaps," Journal of the Aeronautical Sciences, Vol. 22, No. 9, September 1955, pp. 655-656.

${ }^{31}$ Glauert, H., "Theoretical Relationships for an Aerofoil with a Hinged Flap," R \& M No. 1095, British A.R.C., 1927.

${ }^{32}$ Houghton, E. L., and Carpenter, P. W., Aerodynamics for Engineering Students, $4^{\text {th }}$ Edition, Halsted Press, pp. $251-253$.

${ }^{33}$ Allen, H. J., "Calculation of the Chordwise Load Distribution over Airfoil Sections with Plain, Split, or Serially Hinged Trailing-Edge Flaps,” NACA Report No. 634, 1938.

${ }^{34}$ Glauert, H., The Elements of Aerofoil and Airscrew Theory, $2^{\text {nd }}$ Edition, Cambridge University Press, 1947.

${ }^{35}$ Allen, H. J., "General Theory of Airfoil Sections Having Arbitrary Shape or Pressure Distribution," NACA Report No. 833, 1943.

${ }^{36}$ Neumark, S., "Pressure Distribution on an Airfoil in Nonuniform Motion," Journal of the Aeronautical Sciences, Vol. 19, No. 3, March 1952, pp. 214-215.

${ }^{37}$ Sears, W. R., "Operational methods in the Theory of Airfoils in Non-Uniform Motion," Journal of the Franklin Institute, Vol. 130, July 1940, pp. 95-111.

${ }^{38}$ Bisplinghoff, R. L., Ashley, H., and Halfman, R. L., Aeroelasticity, Addison-Wesley, 1955, pp. 814-816.

${ }^{39}$ Jones, R. T., "The Unsteady Lift of a Wing of Finite Aspect Ratio," NACA Report No. 681, 1940.

${ }^{40}$ Garrick, I. E., "On Some Reciprocal Relations in the Theory of Non-Stationary Flows,” NACA Report No. $629,1938$.

${ }^{41}$ Yates, G. T., "Optimum Pitching Axes in Flapping Wing Propulsion," Journal of Theoretical Biology, Vol. 120, 1986, pp. 255276.
} 